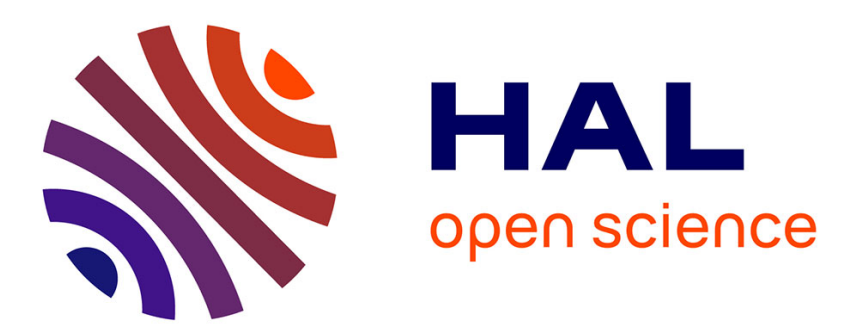

\title{
Facteurs influençant l'intérêt des patients pour l'éducation thérapeutique dans les rhumatismes inflammatoires chroniques
}

Lisa Reyes-Rivet

\section{- To cite this version:}

Lisa Reyes-Rivet. Facteurs influençant l'intérêt des patients pour l'éducation thérapeutique dans les rhumatismes inflammatoires chroniques. Sciences du Vivant [q-bio]. 2020. dumas-03016763

\section{HAL Id: dumas-03016763 https://dumas.ccsd.cnrs.fr/dumas-03016763}

Submitted on 20 Nov 2020

HAL is a multi-disciplinary open access archive for the deposit and dissemination of scientific research documents, whether they are published or not. The documents may come from teaching and research institutions in France or abroad, or from public or private research centers.
L'archive ouverte pluridisciplinaire $\mathbf{H A L}$, est destinée au dépôt et à la diffusion de documents scientifiques de niveau recherche, publiés ou non, émanant des établissements d'enseignement et de recherche français ou étrangers, des laboratoires publics ou privés. 
Facteurs influençant l'intérêt des patients pour l'éducation thérapeutique dans les rhumatismes inflammatoires chroniques

\section{T H E S S E E A R T I C L E}

Présentée et publiquement soutenue devant

LA FACULTÉ DES SCIENCES MEDICALES ET PARAMEDICALES

DE MARSEILLE

Le 20 Octobre 2020

Par Madame Lisa REYES-RIVET

Née le 22 avril 1992 à Annecy (74)

Pour obtenir le grade de Docteur en Médecine

D.E.S. de RHUMATOLOGIE

Membres du Jury de la Thèse :

Monsieur le Professeur LAFFORGUE Pierre

Président

Madame le Professeur PHAM Thao

Assesseur

Monsieur le Professeur ROUDIER Jean

Assesseur

Madame le Docteur TRIJAU Sophie

Assesseur 


\section{FACULTÉ DES SCIENCES MÉDICALES \& PARAMÉDICALES}

\section{Doyen}

Vice-Doyen aux affaires générales

Vice-Doyen aux professions paramédicales

Conseiller

\section{Pr. Georges LEONETTI}

Pr. Patrick DESSI

Pr. Philippe BERBIS

Pr. Patrick VILLANI

\section{Assesseurs :}

aux études

$>$ à la recherche

à l'unité mixte de formation continue en santé

$>$ pour le secteur NORD

> Groupements Hospitaliers de territoire aux masters
Pr. Kathia CHAUMOITRE

Pr. Jean-Louis MEGE

Pr. Justin MICHEL

Pr. Stéphane BERDAH

Pr. Jean-Noël ARGENSON

Pr. Pascal ADALIAN

\section{Chargés de mission :}

sciences humaines et sociales

$>$ relations internationales

$>$ DU/DIU

- DPC, disciplines médicales \& biologiques

$>$ DPC, disciplines chirurgicales

$\begin{array}{ll}: & \text { Pr. Pierre LE COZ } \\ : & \text { Pr. Stéphane RANQUE } \\ : & \text { Pr. Véronique VITTON } \\ : & \text { Pr. Frédéric CASTINETTI } \\ : & \text { Dr. Thomas GRAILLON }\end{array}$

: $\quad$ Pr. Pierre LE COZ

Pr. Véronique VITTON

Dr. Thomas GRAILLON

\section{ÉCOLE DE MEDECINE}

\section{Directeur}

\section{Chargés de mission}

- PACES - Post-PACES

- DFGSM

- DFASM

- DFASM

- Préparation aux ECN

- DES spécialités

- DES stages hospitaliers

- DESMG

- Démographie médicale

- Etudiant

\section{Pr. Jean-Michel VITON}

$\begin{array}{ll}: & \text { Pr. Régis GUIEU } \\ : & \text { Pr. Anne-Laure PELISSIER } \\ : & \text { Pr. Marie-Aleth RICHARD } \\ : \quad \text { Pr. Marc BARTHET } \\ \text { Dr Aurélie DAUMAS } \\ : \quad \text { Pr. Pierre-Edouard FOURNIER } \\ \text { Pr. Benjamin BLONDEL } \\ \text { Pr. Christophe BARTOLI } \\ : \quad \text { Dr. Noémie RESSEGUIER } \\ : \quad \text { Elise DOMINJON }\end{array}$




\section{ÉCOLE DE DE MAIEUTIQUE}

\begin{tabular}{|c|c|c|}
\hline Directrice & : & Madame Carole ZAKARIAN \\
\hline Chargés de mission & & \\
\hline $\begin{array}{l}\text { - } 1^{\mathrm{e}} \text { cycle } \\
\text { - } 2^{\mathrm{e} e n e} \text { cycle }\end{array}$ & $:$ & $\begin{array}{l}\text { Madame Estelle BOISSIER } \\
\text { Madame Cécile NINA }\end{array}$ \\
\hline
\end{tabular}

\section{ÉCOLE DES SCIENCES DE LA RÉADAPTATION}

Directeur

\section{Chargés de mission}

- Masso-kinésithérapie $1^{\text {er }}$ cycle

- Masso-kinésithérapie $2^{\mathrm{e} m e}$ cycle

- Mutualisation des enseignements
Monsieur Philippe SAUVAGEON

Madame Béatrice CAORS

Mada me Joannie HENRY

Madame Géraldine DEPRES

\section{ÉCOLE DES SCIENCES INFIRMIERES}

\section{Directeur}

\section{Chargés de mission}

- Chargée de mission

- Chargé de mission

\section{Monsieur Sébastien COLSON}

Madame Sandrine MAYEN RODRIGUES

Monsieur Christophe ROMAN 


\section{PROFESSEURS HONORAIRES}

MM AGOSTINI Serge ALDIGHIERI René ALESSANDRINI Pierre

ALLIEZ Bernard AQUARON Robert ARGEME Maxime ASSADOURIAN Robert AUFFRAY Jean-Pierre AUTILLO-TOUATI Amapola AZORIN Jean-Michel

BAILLE Yves BARDOT Jacques BARDOT André BERARD Pierre BERGOIN Maurice BERLAND Yvon BERNARD Dominique BERNARD Jean-Louis BERNARD Pierre-Marie BERTRAND Edmond BISSET Jean-Pierre BLANC Bernard BLANC Jean-Louis BOLLINI Gérard BONGRAND Pierre BONNEAU Henri BONNOIT Jean BORY Michel BOTTA Alain BOURGEADE Augustin BOUVENOT Gilles BOUYALA Jean-Marie BREMOND Georges BRICOT René BRUNET Christian BUREAU Henri CAMBOULIVES Jean CANNONI Maurice CARTOUZOU Guy CAU Pierre CHABOT Jean-Michel CHAMLIAN Albert CHARPIN Denis CHARREL Michel CHAUVEL Patrick CHOUX Maurice CIANFARANI François CLAVERIE Jean-Michel CLEMENT Robert COMBALBERT André CONTE-DEVOLX Bernard CORRIOL Jacques COULANGE Christian DALMAS Henri DE MICO Philippe DESSEIN Alain DELARQUE Alain DEVIN Robert DEVRED Philippe DJIANE Pierre DONNET Vincent DUCASSOU Jacques
MM DUFOUR Michel

DUMON Henri ENJALBERT Alain

FAVRE Roger

FIECHI Marius

FARNARIER Georges

FIGARELLA Jacques

FONTES Michel

FRANCES Yves

FRANCOIS Georges

FUENTES Pierre

GABRIEL Bernard

GALINIER Louis

GALLAIS Hervé

GAMERRE Marc

GARCIN Michel

GARNIER Jean-Marc

GAUTHIER André

GERARD Raymond

GEROLAMI-SANTANDREA André

GIUDICELLI Roger

GIUDICELLI Sébastien

GOUDARD Alain

GOUIN François

GRILLO Jean-Marie

GRISOLI François

GROULIER Pierre

HADIDA/SAYAG Jacqueline

HASSOUN Jacques

HEIM Marc

HOUEL Jean

HUGUET Jean-François

JAQUET Philippe

JAMMES Yves

JOUVE Paulette

JUHAN Claude

JUIN Pierre

KAPHAN Gérard

KASBARIAN Michel

KLEISBAUER Jean-Pierre

LACHARD Jean

LAFFARGUE Pierre

LAUGIER René

LE TREUT Yves

LEVY Samuel

LOUCHET Edmond

LOUIS René

LUCIANI Jean-Marie

MAGALON Guy

MAGNAN Jacques

MALLAN-MANCINI Josette

MALMEJAC Claude

MARANINCHI Dominique

MARTIN Claude

MATTEI Jean François

MERCIER Claude

METGE Paul

MICHOTEY Georges

MIRANDA François

MONFORT Gérard

MONGES André

MONGIN Maurice 
PROFESSEURS HONORAIRES

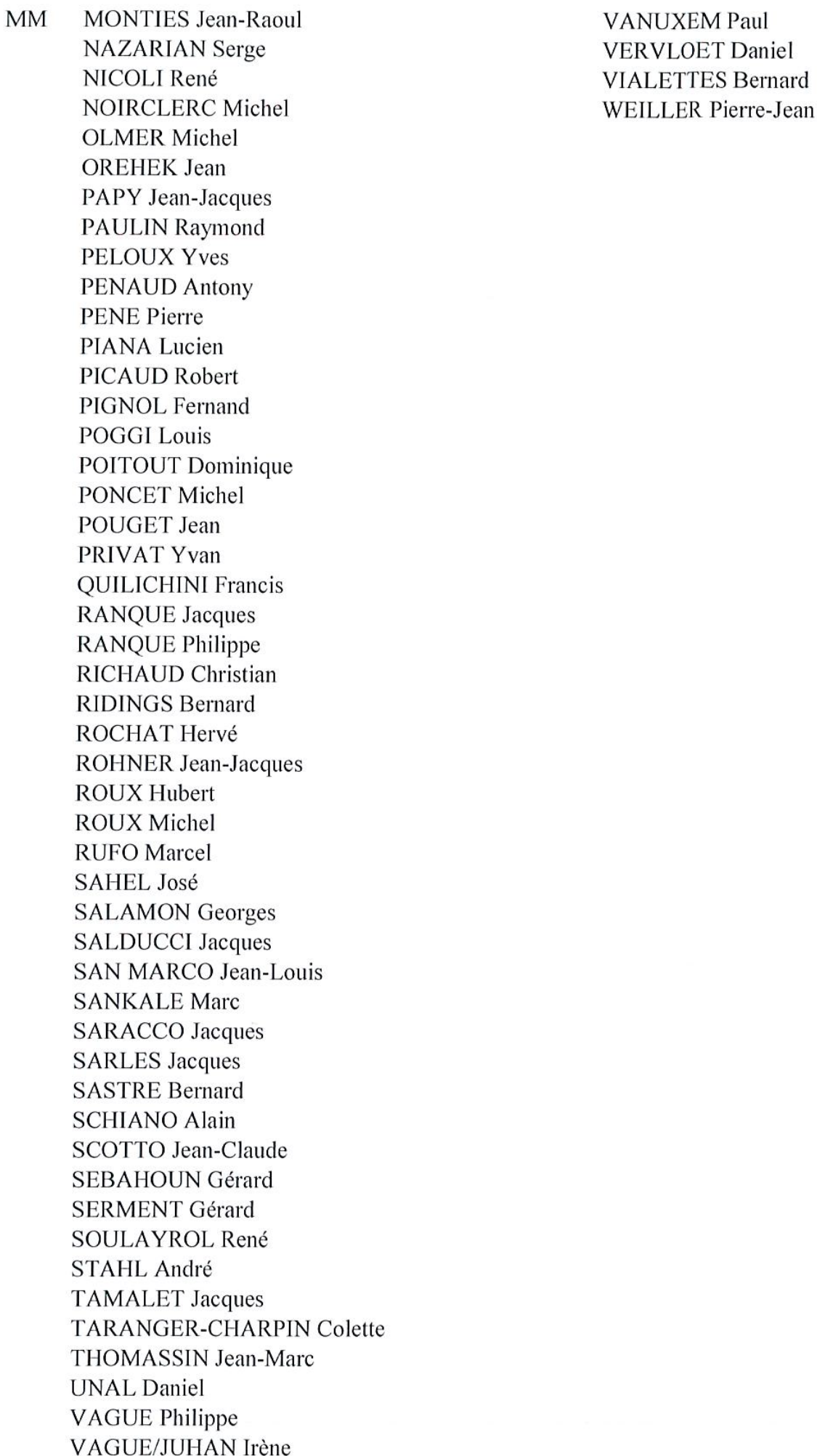


M. le Professeur Mme le Professeur M. le Professeur M. le Professeur M. le Professeur 2009

M. le Professeur M. le Professeur 2010

M. le Professeur 2011

M. le Professeur M. le Professeur M. le Professeur

\section{2}

M. le Professeur M. le Professeur M. le Professeur M. le Professeur M. le Professeur M. le Professeur M. le Professeur

\section{3}

M. le Professeur M. le Professeur M. le Professeur M. le Professeur M. le Professeur M. le Professeur M. le Professeur M. le Professeur

\section{4}

M. le Professeur M. le Professeur M. le Professeur M. le Professeur M. le Professeur

\section{5}

M. le Professeur M. le Professeur M. le Professeur M. le Professeur M. le Professeur M. le Professeur
LEVY Samuel

$31 / 08 / 2011$

JUHAN-VAGUE Irène $\quad 31 / 08 / 2011$

PONCET Michel 31/08/2011

KASBARIAN Michel 31/08/2011

ROBERTOUX Pierre 31/08/2011

DJIANE Pierre

VERVLOET Daniel

$31 / 08 / 2011$

$31 / 08 / 2012$

MAGNAN Jacques

$31 / 12 / 2014$

DI MARINO Vincent

$31 / 08 / 2015$

MARTIN Pierre

$31 / 08 / 2015$

METRAS Dominique

$31 / 08 / 2015$

AUBANIAC Jean-Manuel 31/08/2015

BOUVENOT Gilles $\quad 31 / 08 / 2015$

CAMBOULIVES Jean $\quad 31 / 08 / 2015$

FAVRE Roger $\quad 31 / 08 / 2015$

MATTEI Jean-François $\quad 31 / 08 / 2015$

OLIVER Charles $\quad 31 / 08 / 2015$

VERVLOET Daniel 31/08/2015

BRANCHEREAU Alain 31/08/2016

CARAYON Pierre $\quad 31 / 08 / 2016$

COZZONE Patrick 31/08/2016

DELMONT Jean $\quad 31 / 08 / 2016$

HENRY Jean-François $\quad 31 / 08 / 2016$

LE GUICHAOUA Marie-Roberte 31/08/2016

RUFO Marcel 31/08/2016

SEBAHOUN Gérard 31/08/2016

FUENTES Pierre $\quad 31 / 08 / 2017$

GAMERRE Marc 31/08/2017

MAGALON Guy $\quad 31 / 08 / 2017$

PERAGUT Jean-Claude $\quad 31 / 08 / 2017$

WEILLER Pierre-Jean 31/08/2017

COULANGE Christian $\quad 31 / 08 / 2018$

COURAND François $\quad 31 / 08 / 2018$

FAVRE Roger $\quad 31 / 08 / 2016$

MATTEI Jean-François $\quad 31 / 08 / 2016$

OLIVER Charles $\quad 31 / 08 / 2016$

VERVLOET Daniel $\quad 31 / 08 / 2016$ 
EMERITAT

2016

M. le Professeur

BONGRAND Pierre

$31 / 08 / 2019$

M. le Professeur BOUVENOT Gilles

$31 / 08 / 2017$

M. le Professeur

BRUNET Christian

$31 / 08 / 2019$

CAU Pierre

$31 / 08 / 2019$

COZZONE Patrick 31/08/2017

FAVRE Roger $\quad 31 / 08 / 2017$

FONTES Michel 31/08/2019

JAMMES Yves $\quad 31 / 08 / 2019$

NAZARIAN Serge $\quad 31 / 08 / 2019$

OLIVER Charles $\quad 31 / 08 / 2017$

POITOUT Dominique $\quad 31 / 08 / 2019$

SEBAHOUN Gérard 31/08/2017

VIALETTES Bernard $\quad 31 / 08 / 2019$

2017

M. le Professeur

M. le Professeur

ALESSANDRINI Pierre

$31 / 08 / 2020$

BOUVENOT Gilles

$31 / 08 / 2018$

CHAUVEL Patrick

$31 / 08 / 2020$

COZZONE Pierre

$31 / 08 / 2018$

DELMONT Jean

$31 / 08 / 2018$

FAVRE Roger

$31 / 08 / 2018$

M. le Professeur

OLIVER Charles

$31 / 08 / 2018$

SEBBAHOUN Gérard

$31 / 08 / 2018$

2018

M. le Professeur

M. le Professeur

M. le Professeur

M. le Professeur

M. le Professeur

M. le Professeur

MARANINCHI Dominique

$31 / 08 / 2021$

BOUVENOT Gilles $\quad 31 / 08 / 2019$

COZZONE Pierre 31/08/2019

DELMONT Jean $\quad 31 / 08 / 2019$

FAVRE Roger $\quad 31 / 08 / 2019$

OLIVER Charles $\quad 31 / 08 / 2019$

2019

M. le Professeur

M. le Professeur

M. le Professeur

M. le Professeur

M. le Professeur

M. le Professeur

M. le Professeur

M. le Professeur

M. le Professeur

M. le Professeur

M. le Professeur

M. le Professeur

M. le Professeur

BERLAND Yvon
CHARPIN Denis
CLAVERIE Jean-Michel
FRANCES Yves
CAU Pierre
COZZONE Patrick
DELMONT Jean
FAVRE Roger
FONTES Michel
MAGALON Guy
NAZARIAN Serge
OLIVER Charles
WEILLER Pierre-Jean

$31 / 08 / 2022$

$31 / 08 / 2022$

$31 / 08 / 2022$

$31 / 08 / 2022$

$31 / 08 / 2020$

$31 / 08 / 2020$

$31 / 08 / 2020$

$31 / 08 / 2020$

$31 / 08 / 2020$

$31 / 08 / 2020$

$31 / 08 / 2020$

$31 / 08 / 2020$

$31 / 08 / 2020$ 


\section{PROFESSEURS DES UNIVERSITES-PRATICIENS HOSPITALIERS}

AGOSTINI FERRANDES Aubert

ALBANESE Jacques

ALIMI Yves

AMABILE Philippe

AMBROSI Pierre

ANDRE Nicolas

ARGENSON Jean-Noël

ASTOUL Philippe

ATTARIAN Shahram

AUDOUIN Bertrand

AUQUIER Pascal

AVIERINOS Jean-François

AZULAY Jean-Philippe

BAILLY Daniel

BARLESI Fabrice

BARLIER-SETTI Anne

BARTHET Marc

BARTOLI Christophe

BARTOLI Jean-Michel

BARTOLI Michel

BARTOLOMEI Fabrice

BASTIDE Cyrille

BENSOUSSAN Laurent

BERBIS Philippe

BERBIS Julie

BERDAH Stéphane

BERNARD Jean-Paul Retraite au 25/11/2019

BEROUD Christophe

BERTUCCI François

BLAISE Didier

BLIN Olivier

BLONDEL Benjamin

BONIN/GUILLAUME Sylvie

BONELLO Laurent

BONNET Jean-Louis

BOTTA/FRIDLUND Danielle Surnombre

BOUBLI Léon Surnombre

BOUFI Mourad

BOYER Laurent

BREGEON Fabienne

BRETELLE Florence

BROUQUI Philippe

BRUDER Nicolas

BRUE Thierry

BRUNET Philippe

BURTEY Stéphane

CARCOPINO-TUSOLI Xavier

CASANOVA Dominique

CASTINETTI Frédéric

CECCALDI Mathieu

CHAGNAUD Christophe

CHAMBOST Hervé

CHAMPSAUR Pierre

CHANEZ Pascal

CHARAFFE-JAUFFRET Emmanuelle

CHARREL Rémi

CHAUMOITRE Kathia

CHIARONI Jacques

CHINOT Olivier
CHOSSEGROS Cyrille

COLLART Frédéric

COSTELLO Régis

COURBIERE Blandine

COWEN Didier

CRAVELLO Ludovic

CUISSET Thomas

CURVALE Georges Surnombre

DA FONSECA David

DAHAN-ALCARAZ Laetitia

DANIEL Laurent

DARMON Patrice

D'ERCOLE Claude

D'JOURNO Xavier

DEHARO Jean-Claude

DELAPORTE Emmanuel

DELPERO Jean-Robert Surnombre

DENIS Danièle

DISDIER Patrick

DODDOLI Christophe

DRANCOURT Michel

DUBUS Jean-Christophe

DUFFAUD Florence

DUFOUR Henry

DURAND Jean-Marc

DUSSOL Bertrand

EBBO Mikaël

EUSEBIO Alexandre

FAKHRY Nicolas

FAUGERE Gérard Surnombre

FELICIAN Olvier

FENOLLAR Florence

FIGARELLA/BRANGER Dominique

FLECHER Xavier

FOURNIER Pierre-Edouard

FRANCESCHI Frédéric

FUENTES Stéphane

GABERT Jean

GABORIT Bénédicte

GAINNIER Marc

GARCIA Stéphane

GARIBOLDI Vlad

GAUDART Jean

GAUDY-MARQUESTE Caroline

GENTILE Stéphanie

GERBEAUX Patrick

GEROLAMI/SANTANDREA René

GILBERT/ALESSI Marie-Christine

GIORGI Roch

GIOVANNI Antoine

GIRARD Nadine

GIRAUD/CHABROL Brigitte

GONCALVES Anthony

GRANEL/REY Brigitte

GRANVAL Philippe

GREILLIER Laurent

GRIMAUD Jean-Charles

GROB Jean-Jacques
GUEDJ Eric

GUIEU Régis

GUIS Sandrine

GUYE Maxime

GUYOT Laurent

GUYS Jean-Michel Surnombre

HABIB Gilbert

HARDWIGSEN Jean

HARLE Jean-Robert

HOFFART Louis Disponibilité

HOUVENAEGHEL Gilles

JACQUIER Alexis

JOURDE-CHICHE Noémie

JOUVE Jean-Luc

KAPLANSKI Gilles

KARSENTY Gilles

KERBAUL François détachement

KRAHN Martin

LAFFORGUE Pierre

LAGIER Jean-Christophe

LAMBAUDIE Eric

LANCON Christophe

LA SCOLA Bernard

LAUNAY Franck

LAVIEILLE Jean-Pierre

LE CORROLLER Thomas

LECHEVALLIER Eric

LEGRE Régis

LEHUCHER-MICHEL Marie-Pascale

LEONE Marc

LEONETTI Georges

LEPIDI Hubert

LEVY Nicolas

MACE LoÏc

MAGNAN Pierre-Edouard

MANCINI Julien

MATONTI Frédéric Disponibilité

MEGE Jean-Louis

MERROT Thierry

METZLER/GUILLEMAIN Catherine

MEYER/DUTOUR Anne

MICCALEF/ROLL Joëlle

MICHEL Fabrice

MICHEL Gérard

MICHEL Justin

MICHELET Pierre

MILH Mathieu

MILLION Matthieu

MOAL Valérie

MORANGE Pierre-Emmanuel

MOULIN GuY

MOUTARDIER Vincent

MUNDLER Olivier Surnombre

NAUDIN Jean

NICOLAS DE LAMBALLERIE Xavier

NICOLLAS Richard

OLIVE Daniel

OUAFIK L'Houcine

OVAERT-REGGIO Caroline 


\title{
PROFESSEURS DES UNIVERSITES-PRATICIENS HOSPITALIERS
}

PAGANELLI Franck

PANUEL Michel

PAPAZIAN Laurent

PAROLA Philippe

PARRATTE Sébastien Disponibilité

PELISSIER-ALICOT Anne-Laure

PELLETIER Jean

PERRIN Jeanne

PETIT Philippe

PHAM Thao

PIERCECCHI/MARTI Marie-Dominique

PIQUET Philippe

PIRRO Nicolas

POINSO François

RACCAH Denis

RANQUE Stéphane

RAOULT Didier

REGIS Jean

REYNAUD/GAUBERT Martine

REYNAUD Rachel

RICHARD/LALLEMAND Marie-Aleth

ROCHE Pierre-Hugues
ROCH Antoine

ROCHWERGER Richard

ROLL Patrice

ROSSI Dominique

ROSSI Pascal

ROUDIER Jean

SALAS Sébastien

SAMBUC Roland Surnombre

SARLES/PHILIP Nicole

SARLON-BARTOLI Gabrielle

SCAVARDA Didier

SCHLEINITZ Nicolas

SEBAG Frédéric

SEITZ Jean-François

SIELEZNEFF Igor

SIMON Nicolas

STEIN Andréas

TAIEB David

THIRION Xavier

THOMAS Pascal

THUNY Franck

TREBUCHON-DA FONSECA Agnès
TRIGLIA Jean-Michel TROPIANO Patrick TSIMARATOS Michel TURRINI Olivier

VALERO René

VAROQUAUX Arthur Damien VELLY Lionel

VEY Norbert

VIDAL Vincent

VIENS Patrice

VILLANI Patrick

VITON Jean-Michel

VITTON Véronique

VIEHWEGER Heide Elke

VIVIER Eric

XERRI Luc

\section{PROFESSEUR DES UNIVERSITES}

\author{
ADALIAN Pascal \\ AGHABABIAN Valérie \\ BELIN Pascal \\ CHABANNON Christian \\ CHABRIERE Eric \\ FERON François \\ LE COZ Pierre \\ LEVASSEUR Anthony \\ RANJEVA Jean-Philippe \\ SOBOL Hagay \\ PROFESSEUR CERTIFIE
}

BRANDENBURGER Chantal

PRAG

TANTI-HARDOUIN Nicolas

PROFESSEUR DES UNIVERSITES MEDECINE GENERALE

GENTILE Gaëtan

PROFESSEUR ASSOCIE DE MEDECINE GENERALE $A$ MI-TEMPS

ADNOT Sébastien

GUIDA Pierre

PROFESSEUR ASSOCIE DES UNIVERSITES (disciplines médicales) 
MAITRES DE CONFERENCES DES UNIVERSITES-PRATICIENS HOSPITALIERS

AHERFI Sarah ELDIN Carole

ANGELAKIS Emmanouil (disponibilité) FABRE Alexandre

ATLAN Catherine (disponibilité)

FAURE Alice

BARTHELEMY Pierre

BEGE Thierry

BELIARD Sophie

BENYAMINE Audrey

BERGE-LEFRANC Jean-Louis

BERTRAND Baptiste

BEYER-BERJOT Laura

BIRNBAUM David

BONINI Francesca

BOUCRAUT Joseph

BOULAMERY Audrey

BOULLU/CIOCCA Sandrine

BOUSSEN Salah Michel

BUFFAT Christophe

CAMILLERI Serge

CARRON Romain

CASSAGNE Carole

CERMOLACCE Michel

CHAUDET Hervé

CHRETIEN Anne-Sophie

COZE Carole

CUNY Thomas

DADOUN Frédéric (disponibilité)

DALES Jean-Philippe

DAUMAS Aurélie

DEGEORGES/VITTE Joëlle

DELLIAUX Stéphane

DESPLAT/JEGO Sophie

DEVILLIER Raynier

DUBOURG Grégory

DUCONSEIL Pauline

DUFOUR Jean-Charles
NINOVE Laetitia

NOUGAIREDE Antoine

OLLIVIER Matthieu

PAULMYER/LACROIX Odile

PESENTI Sébastien

RADULESCO Thomas

RESSEGUIER Noémie

ROBERT Philippe

ROMANET Pauline

SABATIER Renaud

SARI-MINODIER Irène

SAVEANU Alexandru

SECQ Véronique (disponibilité)

STELLMANN Jan-Patrick

SUCHON Pierre

TABOURET Emeline

TOGA Caroline

TOGA Isabelle

TOMASINI Pascale

TOSELLO Barthélémy

TROUSSE Delphine

TUCHTAN-TORRENTS Lucile

VELY Frédéric

VION-DURY Jean

ZATTARA/CANNONI Hélène

\section{MAITRES DE CONFERENCES DES UNIVERSITES}

(mono-appartenants)

ABU ZAINEH Mohammad

BARBACARU/PERLES T. A.

BERLAND/BENHAIM Caroline BOUCAULT/GARROUSTE Françoise BOYER Sylvie

COLSON Sébastien
DEGIOANNI/SALLE Anna

DESNUES Benoît

MARANINCHI Marie

MERHEJ/CHAUVEAU Vicky

MINVIELLE/DEVICTOR Bénédicte

POGGI Marjoric
POUGET Benoît

RUEL Jérôme

THOLLON Lionel

THIRION Sylvie

VERNA Emeline

MAITRE DE CONFERENCES DES UNIVERSITES DE MEDECINE GENERALE

CASANOVA Ludovic

MAITRES DE CONFERENCES ASSOCIES DE MEDECINE GENERALE à MI-TEMPS

BARGIER Jacques

BONNET Pierre-André

CALVET-MONTREDON Céline

JANCZEWSKI Aurélie

NUSSLI Nicolas

ROUSSEAU-DURAND Raphaëlle

THERY Didier (nomination au 1/10/2019) 
MAITRE DE CONFERENCES ASSOCIE à MI-TEMPS

BOURRIQUEN Maryline

EVANS-VIALLAT Catherine

LUCAS Guillaume

MATHIEU Marion

MAYENS-RODRIGUES Sandrine

MELLINAS Marie

REVIS Joana

ROMAN Christophe

TRINQUET Laure 


\section{PROFESSEURS DES UNIVERSITES et MAITRES DE CONFERENCES DES UNIVERSITES - PRATICIENS HOSPITALIERS PROFESSEURS ASSOCIES, MAITRES DE CONFERENCES DES UNIVERSITES mono-appartenants}

\begin{tabular}{|c|c|}
\hline ANATOMIE 4201 & ANTHROPOLOGIE 20 \\
\hline CHAMPSAUR Pierre (PU-PH) & ADALIAN Pascal (PR) \\
\hline \multicolumn{2}{|l|}{ LE CORROLLER Thomas (PU-PH) } \\
\hline \multirow[t]{3}{*}{ PIRRO Nicolas (PU-PH) } & DEGIOANNI/SALLE Anna (MCF) \\
\hline & POUGET Benoît (MCF) \\
\hline & VERNA Emeline (MCF) \\
\hline \multicolumn{2}{|l|}{ GUENOUN-MEYSSIGNAC Daphné (MCU-PH) } \\
\hline LAGIER Aude (MCU-PH) disponibilité & BACTERIOLOGIE-VIROLOGIE ; HYGIENE HOSPITALIERE 4501 \\
\hline \multirow[t]{4}{*}{ THOLLON Lionel (MCF) (60ème section) } & CHARREL Rémi (PU PH) \\
\hline & DRANCOURT Michel (PU-PH) \\
\hline & FENOLLAR Florence (PU-PH) \\
\hline & FOURNIER Pierre-Edouard (PU-PH) \\
\hline \multirow[t]{2}{*}{ ANATOMIE ET CYTOLOGIE PATHOLOGIQUES 4203} & JNICOLAS DE LAMBALLERIE Xavier (PU-PH) \\
\hline & LA SCOLA Bernard (PU-PH) \\
\hline CHARAFE/JAUFFRET Emmanuelle (PU-PH) & RAOULT Didier (PU-PH) \\
\hline \multicolumn{2}{|l|}{ DANIEL Laurent (PU-PH) } \\
\hline FIGARELLA/BRANGER Dominique (PU-PH) & AHERFI Sarah (MCU-PH) \\
\hline GARCIA Stéphane (PU-PH) & ANGELAKIS Emmanouil (MCU-PH) disponibilité \\
\hline \multirow[t]{3}{*}{ XERRI Luc (PU-PH) } & DUBOURG Grégory (MCU-PH) \\
\hline & GOURIET Frédérique (MCU-PH) \\
\hline & NOUGAIREDE Antoine (MCU-PH) \\
\hline DALES Jean-Philippe (MCU-PH) & NINOVE Laetitia (MCU-PH) \\
\hline \multicolumn{2}{|l|}{ GIUSIANO COURCAMBECK Sophie (MCU PH) } \\
\hline LABIT/BOUVIER Corinne (MCU-PH) & CHABRIERE Eric (PR) (64ème section) \\
\hline \multicolumn{2}{|l|}{ MACAGNO Nicolas (MCU-PH) } \\
\hline MAUES DE PAULA André (MCU-PH) & LEVASSEUR Anthony (PR) (64ème section) \\
\hline \multirow[t]{2}{*}{ SECQ Véronique (MCU-PH) disponibilité } & DESNUES Benoit (MCF) ( 65ème section ) \\
\hline & MERHEJ/CHAUVEAU Vicky (MCF) (87ème section) \\
\hline
\end{tabular}

ANESTHESIOLOGIE ET REANIMATION CHIRURGICALE ; MEDECINE URGENCE 4801

ALBANESE Jacques (PU-PH)

BRUDER Nicolas (PU-PH)

LEONE Marc (PU-PH)

MICHEL Fabrice (PU-PH)

VELLY Lionel (PU-PH)

BOUSSEN Salah Michel (MCU-PH)

GUIDON Catherine (MCU-PH)
BIOCHIMIE ET BIOLOGIE MOLECULAIRE 4401

BARLIER/SETTI Anne (PU-PH)

GABERT Jean (PU-PH)

GUIEU Régis (PU-PH)

OUAFIK L'Houcine (PU-PH)

BUFFAT Christophe (MCU-PH)

FROMONOT Julien (MCU-PH)

MOTTOLA GHIGO Giovanna (MCU-PH)

ROMANET Pauline (MCU-PH)

SAVEANU Alexandru (MCU-PH)

\section{ANGLAIS 11}

BRANDENBURGER Chantal (PRCE)
ROLL Patrice (PU-PH)

FRANKEL Diane (MCU-PH)

GASTALDI Marguerite (MCU-PH)

KASPI-PEZZOLI Elise (MCU-PH)

LEVY-MOZZICONNACCI Annie (MCU-PH)

ET DE LA REPRODUCTION ; GYNECOLOGIE MEDICALE 5405

METZLER/GUILLEMAIN Catherine (PU-PH)

PERRIN feanne (PU.PH)

MAJ 01.09.2019 
GUEDJ Eric (PU-PH)

GUYE Maxime (PU-PH)

MUNDLER Olivier (PU-PH) Surnombre

TAIEB David (PU-PH)

BELIN Pascal (PR) (69ème section)

RANJEVA Jean-Philippe (PR) (69ème section)

CAMMILLERI Serge (MCU-PH)

VION-DURY Jean (MCU-PH)

BARBACARU/PERLES Téodora Adriana (MCF) (69ème section)
AVIERINOS Jean-François (PU-PH)

BONELLO Laurent (PUPH)

BONNET Jean-Louis (PU-PH)

CUISSET Thomas (PU-PH)

DEHARO Jean-Claude (PU-PH)

FRANCESCHI Frédéric (PU-PH)

HABIB Gilbert (PU-PH)

PAGANELLI Franck (PU-PH)

THUNY Franck (PU-PH)
BIOSTATISTIQUES, INFORMATIQUE MEDICALE

ET TECHNOLOGIES DE COMMUNICATION 4604
GAUDART Jean (PU-PH)

GIORGI Roch (PU-PH)

MANCINI Julien (PU-PH)

CHAUDET Hervé (MCU-PH)

DUFOUR Jean-Charles (MCU-PH)

GIUSIANO Bernard (MCU-PH)

ABU ZAINEH Mohammad (MCF) (5ème section)

BOYER Sylvie (MCF) (5ème section)

\section{CHIRURGIE VISCERALE ET DIGESTIVE 5202}

BERDAH Stéphane (PU-PH)

DELPERO Jean-Robert (PU-PH) Surnombre

HARDWIGSEN Jean (PU-PH)

MOUTARDIER Vincent (PU-PH)

SEBAG Frédéric (PU-PH)

SIELEZNEFF Igor (PU-PH)

TURRINI Olivier (PU-PH)

BEGE Thierry (MCU-PH)

BEYER-BERJOT Laura (MCU-PH)

BIRNBAUM David (MCU-PH)

DUCONSEIL Pauline (MCU-PH)

GUERIN Carole (MCU PH)

CHIRURGIE ORTHOPEDIQUE ET TRAUMATOLOGIQUE 5002

ARGENSON Jean-Noël (PU-PH)

BLONDEL Benjamin (PU-PH)

CURVALE Georges (PU-PH) Surnombre

FLECHER Xavier (PU PH)

PARRATTE Sébastien (PU-PH) Disponibilité

ROCHWERGER Richard (PU-PH)

TROPIANO Patrick (PU-PH)

OLLIVIER Matthieu (MCU-PH)

\section{CANCEROLOGIE ; RADIOTHERAPIE 4702}

BERTUCCI François (PU-PH)

CHINOT Olivier (PU-PH)

COWEN Didier (PU-PH)

DUFFAUD Florence (PU-PH)

GONCALVES Anthony PU-PH)

HOUVENAEGHEL Gilles (PU-PH)

LAMBAUDIE Eric (PU-PH)

SALAS Sébastien (PU-PH)

VIENS Patrice (PU-PH)

SABATIER Renaud (MCU-PH)

TABOURET Emeline (MCU-PH) 


\section{COLLART Frédéric (PU-PH)}

D'JOURNO Xavier (PU-PH)

DODDOLI Christophe (PU-PH)

GARIBOLDI Vlad (PU-PH)

MACE LoÏc (PU-PH)

THOMAS Pascal (PU-PH)

FOUILLOUX Virginie (MCU-PH)

TROUSSE Delphine (MCU-PH)
CASANOVA Dominique (PU-PH)

LEGRE Régis (PU-PH)

BERTRAND Baptiste (MCU-PH)

HAUTIER/KRAHN Aurélie (MCU-PH)

\section{CHIRURGIE VASCULAIRE ; MEDECINE VASCULAIRE 5104}

ALIMI Yves (PU-PH)

AMABILE Philippe (PU-PH)

GASTROENTEROLOGIE ; HEPATOLOGIE ; ADDICTOLOGIE 5201

BARTOLI Michel (PU-PH)

BOUFI Mourad (PU-PH)

MAGNAN Pierre-Edouard (PU-PH)

PIQUET Philippe (PU-PH)

SARLON-BARTOLI Gabrielle (PU PH)

BERNARD Jean-Paul (PU-PH) retraite au 25/11/2019

BOTTA-FRIDLUND Danielle (PU-PH) Surnombre

DAHAN-ALCARAZ Laetitia (PU-PH)

GEROLAMI-SANTANDREA René (PU-PH)

HISTOLOGIE, EMBRYOLOGIE ET CYTOGENETIQUE 4202

GRANDVAL Philippe (PU-PH)

GRIMAUD Jean-Charles (PU-PH)

LEPIDI Hubert (PU-PH)

SEITZ Jean-François (PU-PH)

LEPIDI Hubert (PU-PH)

VITTON Véronique (PU-PH)

PAULMYER/LACROIX Odile (MCU-PH)

GONZALEZ Jean-Michel ( MCU-PH)

DERMATOLOGIE - VENEREOLOGIE 5003

GENETIQUE 4704

BERBIS Philippe (PU-PH)

DELAPORTE Emmanuel (PU-PH)

GAUDY/MARQUESTE Caroline (PU-PH)

GROB Jean-Jacques (PU-PH)

RICHARD/LALLEMAND Marie-Aleth (PU-PH)

DUSI

COLSON Sébastien (MCF)

MYUEN Karine (MCU-PH)

TOGA Caroline (MCU-PH)

ZATTARA/CANNONI Hélène (MCU-PH)

BOURRIQUEN Maryline (MAST)

EVANS-VIALLAT Catherine (MAST)

LUCAS Guillaume (MAST)

MAYEN-RODRIGUES Sandrine (MAST)

MELLINAS Marie (MAST)

ROMAN Christophe (MAST)

TRINQUET Laure (MAST)

\section{ENDOCRINOLOGIE ,DIABETE ET MALADIES METABOLIQUES ; GYNECOLOGIE MEDICALE 5404}

BRUE Thierry (PU-PH)

CASTINETTI Frédéric (PU-PH)

CUNY Thomas (MCU PH) 
AUQUIER Pascal (PU-PH)

BERBIS Julie (PU-PH)

BOYER Laurent (PU-PH)

GENTILE Stéphanie (PU-PH)

SAMBUC Roland (PU-PH) Surnombre

THIRION Xavier (PU-PH)

LAGOUANELLE/SIMEONI Marie-Claude (MCU-PH)

RESSEGUIER Noémie (MCU-PH)

MINVIELLE/DEVICTOR Bénédicte (MCF)(06ème section)

TANTI-HARDOUIN Nicolas (PRAG)
BLAISE Didier (PU-PH)

COSTELLO Régis (PU-PH)

CHIARONI Jacques (PU-PH)

GILBERT/ALESSI Marie-Christine (PU-PH)

MORANGE Pierre-Emmanuel (PU-PH)

VEY Norbert (PU-PH)

DEVILLIER Raynier (MCU PH)

GELSI/BOYER Véronique (MCU-PH)

LAFAGE/POCHITALOFF-HUVALE Marina (MCU-PH)

LOOSVELD Marie (MCU-PH)

SUCHON Pierre (MCU-PH)

POGGI Marjorie (MCF) (64ème section)
KAPLANSKI Gilles (PU-PH)

MEGE Jean-Louis (PU-PH)

OLIVE Daniel (PU-PH)

VIVIER Eric (PU-PH)

FERON François (PR) (69ème section)

BOUCRAUT Joseph (MCU-PH)

CHRETIEN Anne-Sophie (MCU PH)

DEGEORGES/VITTE Joëlle (MCU-PH)

DESPLAT/JEGO Sophie (MCU-PH)

ROBERT Philippe (MCU-PH)

VELY Frédéric (MCU-PH)

BOUCAULT/GARROUSTE Françoise (MCF) 65ème section)

MEDECINE PHYSIQUE ET DE READAPTATION 4905

MALADIES INFECTIEUSES ; MALADIES TROPICALES 4503

BROUQUI Philippe (PU-PH)

LAGIER Jean-Christophe (PU-PH)

MILLION Matthieu (PU-PH)

PAROLA Philippe (PU-PH)

STEIN Andréas (PU-PH)

ELDIN Carole (MCU-PH)

MEDECINE D'URGENCE 4805

MEDECINE LEGALE ET DROIT DE LA SANTE 4603

BARTOLI Christophe (PU-PH)

LEONETTI Georges (PU-PH)

PELISSIER-ALICOT Anne-Laure (PU-PH)

PIERCECCHI-MARTI Marie-Dominique (PU-PH)

TUCHTAN-TORRENTS Lucile (MCU-PH)

BERLAND/BENHAIM Caroline (MCF) (1ère section)
BENSOUSSAN Laurent (PU-PH)

VITON Jean-Michel (PU-PH)
MEDECINE ET SANTE AU TRAVAIL 4602

LEHUCHER/MICHEL Marie-Pascale (PU-PH)

BERGE-LEFRANC Jean-Louis (MCU-PH)

SARI/MINODIER Irène (MCU-PH)

KERBAUL François (PU-PH) détachement

MICHELET Pierre (PU-PH)

\begin{tabular}{c}
\hline MEDECINE INTERNE ; GERIATRIE ET BIOLOGIE DU \\
VIEILLISSEMENT ; ADDICTOLOGIE 5301 \\
\hline
\end{tabular}

BONIN/GUILLAUME Sylvie (PU-PH)

DISDIER Patrick (PU-PH)

DURAND Jean-Marc (PU-PH)

GRANEL/REY Brigitte (PU-PH)

HARLE Jean-Robert (PU-PH)

ROSSI Pascal (PU-PH)

SCHLEINITZ Nicolas (PU-PH)

BENYAMINE Audrey (MCU-PH)

EBBO Mikepk(MCD-PH)pus Timone 
GENTILE Gaëtan (PR Méd. Gén. Temps plein)

CASANOVA Ludovic (MCF Méd. Gén. Temps plein)

ADNOT Sébastien (PR associé Méd. Gén. à mi-temps)

GUIDA Pierre (PR associé Méd. Gén. à mi-temps)

BARGIER Jacques (MCF associé Méd. Gén. À mi-temps)

BONNET Pierre-André (MCF associé Méd. Gén à mi-temps)

CALVET-MONTREDON Céline (MCF associé Méd. Gén. à temps plein)

JANCZEWSKI Aurélie (MCF associé Méd. Gén. À mi-temps)

NUSSLI Nicolas (MCF associé Méd. Gén. À mi-temps)

ROUSSEAU-DURAND Raphaëlle (MCF associé Méd. Gén. À mi-temps)

THERY Didier (MCF associé Méd. Gén. À mi-temps) (nomination au 1/10/2019)

\section{NUTRITION 4404}

DARMON Patrice (PU-PH)

RACCAH Denis (PU-PH)

VALERO René (PU-PH)

ATLAN Catherine (MCU-PH) disponibilité

BELIARD Sophie (MCU-PH)

MARANINCHI Marie (MCF) (66ème section)
BRUNET Philippe (PU-PH)

BURTEY Stépahne (PU-PH)

DUSSOL Bertrand (PU-PH)

JOURDE CHICHE Noémie (PU PH)

MOAL Valérie (PU-PH)
DUFOUR Henry (PU-PH)

FUENTES Stéphane (PU-PH)

REGIS Jean (PU-PH)

ROCHE Pierre-Hugues (PU-PH)

SCAVARDA Didier (PU-PH)

CARRON Romain (MCU PH)

GRAILLON Thomas (MCU PH) \begin{tabular}{|c|}
\hline ONCOLOGIE 65 (BIOLOGIE CELLULAIRE) \\
\hline
\end{tabular}

CHABANNON Christian (PR) (66ème section)

SOBOL Hagay (PR) (65ème section)

\section{OPHTALMOLOGIE 5502}

DENIS Danièle (PU-PH)

HOFFART Louis (PU-PH) Disponibilité

MATONTI Frédéric (PU-PH) Disponibilité

\section{OTO-RHINO-LARYNGOLOGIE 5501}

\section{DESSI Patrick (PU-PH)}

FAKHRY Nicolas (PU-PH)

GIOVANNI Antoine (PU-PH)

LAVIEILLE Jean-Pierre (PU-PH)

MICHEL Justin (PU-PH)

NICOLLAS Richard (PU-PH)

TRIGLIA Jean-Michel (PU-PH)

RADULESCO Thomas (MCU-PH)

REVIS Joana (MAST) (Orthophonie) (7ème Section)

\section{NEUROLOGIE 4901}

ATTARIAN Sharham (PUPH)

AUDOIN Bertrand (PU-PH)

AZULAY Jean-Philippe (PU-PH)

CECCALDI Mathieu (PU-PH)

EUSEBIO Alexandre (PU-PH)

FELICIAN Olivier (PU-PH)

PELLETIER Jean (PU-PH)

MAAROUF Adil (MCU-PH)

PEDOPSYCHIATRIE; ADDICTOLOGIE 4904

DA FONSECA David (PU-PH)

POINSO François (PU-PH)

GUIVARCH Jokthan (MCU-PH)
BLIN Olivier (PU-PH)

FAUGERE Gérard (PU-PH) Surnombre

MICALLEF/ROLL Joëlle (PU-PH)

SIMON Nicolas (PU-PH)

BOULAMERY Audrey (MCU-PH) 
RANQUE Stéphane (PU-PH)

CASSAGNE Carole (MCU-PH)

L'OLLIVIER Coralie (MCU-PH)

TOGA Isabelle (MCU-PH)
LE COZ Pierre (PR) (17ème section)

MATHIEU Marion (MAST)

PHYSIOLOGIE 4402

BARTOLOMEI Fabrice (PU-PH)

BREGEON Fabienne (PU-PH)

GABORIT Bénédicte (PU-PH)

MEYERIDUTOUR Anne (PU-PH)

TREBUCHON/DA FONSECA Agnès (PU-PH)

BARTHELEMY Pierre (MCU-PH)

BONINI Francesca (MCU-PH)

BOULLU/CIOCCA Sandrine (MCU-PH)

DADOUN Frédéric (MCU-PH) (disponibilité)

DELLIAUX Stéphane (MCU-PH)

RUEL Jérôme (MCF) (69ème section)

THIRION Sylvie (MCF) (66ème section)

PSYCHIATRIE D'ADULTES ; ADDICTOLOGIE 4903

\section{PNEUMOLOGIE; ADDICTOLOGIE 5101}

BAILLY Daniel (PU-PH)

LANCON Christophe (PU-PH)

NAUDIN Jean (PU-PH)

ASTOUL Philippe (PU-PH)

BARLESI Fabrice (PU-PH)

CHANEZ Pascal (PU-PH)

CERMOLACCE Michel (MCU-PH)

GREILLIER Laurent (PU PH)

PSYCHOLOGIE - PSYCHOLOGIE CLINIQUE, PCYCHOLOGIE SOCIALE 16

AGHABABIAN Valérie (PR)

TOMASINI Pascale (MCU-PH)
REYNAUD/GAUBERT Martine (PU-PH)

RADIOLOGIE ET IMAGERIE MEDICALE 4302

BARTOLI Jean-Michel (PU-PH)

CHAGNAUD Christophe (PU-PH)

CHAUMOITRE Kathia (PU-PH)

GIRARD Nadine (PU-PH)

JACQUIER Alexis (PU-PH)

MOULIN Guy (PU-PH)

PANUEL Michel (PU-PH)

PETIT Philippe (PU-PH)

VAROQUAUX Arthur Damien (PU-PH)

VIDAL Vincent (PU-PH)

STELLMANN Jan-Patrick (MCU-PH)

REANIMATION MEDICALE ; MEDECINE URGENCE 4802

GAINNIER Marc (PU-PH)

GERBEAUX Patrick (PU-PH)

PAPAZIAN Laurent (PU-PH)

ROCH Antoine (PU-PH)

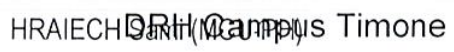

\section{RHUMATOLOGIE 5001}

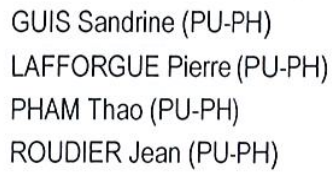

THERAPEUTIQUE; MEDECINE D'URGENCE; ADDICTOLOGIE 4804

AMBROSI Pierre (PU-PH)

VILLANI Patrick (PU-PH)

DAUMAS Aurélie (MCU-PH)

UROLOGIE 5204

BASTIDE Cyrille (PU-PH)

KARSENTY Gilles (PU-PH)

LECHEVALLIER Eric (PU-PH)

ROSSI Dominique (PU-PH)

MAJ 01.09.2019 


\section{REMERCIEMENTS}

\section{Aux membres du Jury,}

A Monsieur le Professeur Lafforgue, président du Jury, Merci de $\mathrm{m}^{\prime}$ avoir fait l'honneur de présider mon jury de thèse. Merci de votre encadrement, de vos enseignements tout au long de mon internat et merci de m'avoir accepté dans votre service pour la suite de ma formation.

A Madame le Professeur Pham, Merci de me faire l'honneur d'être dans mon jury. Merci pour votre investissement dans ma formation, merci pour votre aide précieuse pendant mon internat et particulièrement ces derniers mois, merci pour vos relectures et corrections qui m'ont permis de présenter cette thèse aujourd'hui.

A Monsieur le Pr Roudier,

Merci d'avoir accepté de faire partie de mon jury. Merci tous vos enseignements et pour vos anecdotes en visite.

Au Dr Sophie Trijau, Tout d'abord merci de m'avoir proposé ce sujet de thèse, qui m'a beaucoup intéressé et qui malgré les changements de dernière minute à cause du COVID, a pu voir le jour! Merci pour tout ce que tu m'as appris, j'aimerais beaucoup être une rhumatologue comme toi quand je serais grande.

Au Dr Senbel, merci de m'avoir intégré dans votre programme d'éducation thérapeutique et de $\mathrm{m}^{\prime}$ avoir permis de participer aux ateliers.

Merci aux différents acteurs des programmes d'ETP RIC et Réseau Santé Croisée pour leur aide dans l'élaboration de ma thèse.

\section{A mes parents,}

A mon Papoute, Insupportablement gentil et profondément généreux, sans qui je n'aurais jamais survécu à toutes ces années de médecine. Merci pour $\mathrm{m}^{\prime}$ avoir créé de parfaits petits cocons pour travailler dans les meilleures conditions possibles pendant ces 10 années, d'avoir fait des allers-retours incessants entre Annecy, Grenoble, Marseille...Et d'avoir supporté mon ingratitude...

A ma Moman, A qui je dois tellement de choses..., la joie de vivre, la confiance en moi, le goût des études, et du travail. Être tête en l'air aussi... 
Je ne serais pas là aujourd'hui sans ton soutien, et ton amour inconditionnel, même si je suis souvent insupportable... Je ne pourrais jamais te remercier assez pour tout ce que tu as fait pour moi et pour m'avoir permis de devenir ce que je suis.

A ma Sistouch, le plus cadeau que nos parents ont pu me faire (même si je dis parfois que j'aurai préféré un lapin). Te voir grandir et t'épanouir est une chance et un bonheur. J'espère qu'on pourra se faire des week-ends/semaine sistah plus souvent (prochaine destination les USA ?).

A toute ma famille, Mamie, Nanie, Papy, Papi Chicho, Tonton mouss, tonton Karim, Mina, Chrystèle, Houria, Valérie, Dominique, et tous mes cousins (Marine et Mathilde mes 2 supers cousines, Sophien, Mehdi, Hugo, Martin, Yassine et Nassim, Cindy, et Hayet mes cousines rapportées et mes magnifiques neveux et nièces, Jawed, Savannah et Dania) merci pour votre amour et votre soutien.

A ma Tifmoule, 15 ans d'amitié déjà ou 13 ans suivant à qui on demande à priori... On a grandi ensemble à travers tous les âges, l'âge (très) bête, le lycée, les études supérieures, dans des villes voire des pays différents sans jamais se séparer vraiment. Londres, NYC, que de beaux voyages, j'ai hâte du prochain ;) Merci de $m$ 'avoir soutenue toutes ces années ma moule, j'espère que tu pourras me supporter encore pour de longues années.

Ma Lyd, Que de chemin parcouru depuis le lycée ou tu me toisais du haut de ton $1 \mathrm{~m} 75$ !

Bien contente que tu aies abandonné cette imbécile de Diana pour venir avec moi faire des night au Pop plage, sub clubs et autres endroits très selects d'Annecy.

Merci pour toutes ces soirées à discuter de sujets passionnants, ces voyages pas toujours bien organisés (petit échec post nouvel an à Amsterdam).

Manon ma poulette, La personne qui m'a vu plonger en P1 (oui j'entends mon frigo dans ma tête) et qui m'a soutenu envers et contre tout ! Ces covoiturages pour annecy le soir en chantant macklemore à tue-tête. Merci pour ces superbes weekends à $G G$ en zone non tendue, j'espère pouvoir en faire pleins d'autres!

A Margeounette, Qui m'a permis grâce a ses nombreux erasmus de faire le tour du monde ! Merci de m'avoir accepté à Madrid, en Australie, merci pour cette 
incroyable aventure de 2 semaines dans le coffre d'une voiture, c'était quand même pas banal!

\section{A mes copines de Grenoble,}

Ma july, best first coloc ever! Merci de m'avoir tiré vers le haut pendant ces 4 ans de colocs (mon classement c'est à toi que je le dois !), merci pour ces soirées BU mais surtout twilight, Bachelor, AEDLP...Merci pour l'honneur de $\mathrm{m}$ 'avoir demandé de parler à ton mariage.

Mon Janno, merci pour ces années d'externat, pour ton soutien, ta gentillesse, et ta patience. Pour avoir été notre mère a toutes, pour ton appartement qui nous rassemblait en toutes occasions (soirée sushi, nems, gâteaux, Noel, Nouvel An...), pour les semaines de ski à Bourg, pour cet incroyable voyage dans ta famille au Québec!

Eva, Dr Roule Ta Boule, ma $1^{\text {ère }}$ amie de médecine, rencontrée à la 1ere seconde du $1^{\text {er }}$ tutorat de la P1, et restée mon amie depuis... Même si on se voit moins souvent, tu peux compter sur moi et je sais que l'inverse est vrai aussi. Merci pour ton humour incroyable, et merci d'avoir eu l'idée et d'avoir accepté de faire un sketch avec moi au mariage de Julie, ce fut un franc succès.

Ma Camouille, la plus gentille, la plus douce...de jour ! Et la plus grosse fêtarde de nuit, même si tu t'es un peu assagie récemment (ou est-ce à cause de ce semestre confiné... ?)

Merci pour années d'externat et même d'internat, ces soirées karaokés, et les soirées berthoms-Barberousse qui finissent parfois mal... Merci aussi pour tes talents d'actrice, tu as fait un parfait Siméon!

Cécél, même si tu es toujours en vadrouille, et qu'on a bien du mal à te mettre la main dessus, je suis très heureuse de t'avoir eu comme amie pendant ces années de médecine, tu nous auras bien fait rire avec tes mimes improbables et tes célinades! J'espère que tes voyages t'amèneront à Marseille un de ces 4 !

Mon Paulet, ma grosse paulette, merci pour ces merveilleuses soirées et surtout lendemain de soirée d'externat à loquer sur votre canapé, et à regarder des merveilles télévisuelles, et ces petits déj et café interminables à l'hôpital pendant le stage!

Merci de comprendre toutes mes références, parce qu'on a eu la même enfance (\#le cygne et la princesse). 
Sab, merci pour ces années d'externat, pour ta gentillesse, cette soirée mémorable à boire des litres de vin avec des micro tapas.

\section{La coloc du love,}

Elsa, L'Elson, Zonzon, Zonzi, mon Zonz, le trio de la mort en 1ere année d'internat puis le quatuor du love ensuite, la teufeuse du groupe, la romanichelle de la coloc, la meuf la plus brillante mais aussi la plus chill, je suis trop heureuse de $t^{\prime}$ avoir rencontré, et de t'avoir fait aimé le fromage, $c^{\prime}$ est un grand accomplissement pour moi, peut-être même plus que la thèse... Merci pour ces 4 dernières années et pour j'espère pleins d'autres.

Josette, mon Josy, j'aurai pu te mettre dans les meufs de Grenoble mais bon tu as plus ta place ici, même si nos soirées d'externat avec le Paulet étaient bien mémorables!

La cheffe, la maman de la coloc, toujours présente quand j'ai besoin de toi, même pour les trucs très chiants (je sais que tu serais allé à ma réunion de copro...) merci d'être ma voisine et de m'accueillir à toute heure du jour et de la nuit...

Nini, ma Nins, le bébé de la coloc mais aussi la meilleure cuisinière, qui nous a nourri pendant 2 ans, et c'était pas rien quand on voit les morfals... Merci pour ta joie de vivre, ton dynamisme en toutes circonstances, ta gentillesse et surtout nos blagues OSS !

Je n'arrive pas à déterminer quand a commencé notre amitié mais j'ai l'impression que ça fait depuis toujours... J'espère pouvoir faire encore pleins de voyages incroyables avec toi, manger pleins de fromage, faire du scooter, du yoga, mais pas du trail stp...

Marion, coloc du colloque pendant seulement 6 mois et pourtant tu t'es intégré aussitôt à notre groupe et tu n'en es pas sorti (tu m'as fait venir à Amiens quand même !), j'aurais aimé que tu sois là mais ne crois pas t'en tirer à si bon compte, nous viendrons jusqu'à toi !

Mon Julo,

La $3^{\mathrm{e}}$ du trio LEJ, toi et ton accent du lot, vous êtes incroyable ! Merci pour cette $1^{\text {ère }}$ année $d^{\prime}$ internat, où on régnait sur le $3^{\mathrm{e}}$ étage, merci pour nos brunchs maison toutes les semaines qui m'ont fait tenir cet été, pour notre week-end prolongé en Italie ou on s'en est mis plein la panse et où tu as failli me faire le coup de la panne... 
Mon Soso, Je me rappelle très bien notre 1ere rencontre au trolley bus où tout le monde dansait et que tu tentais d'avoir une conversation profonde avec moi au milieu de la piste... ça te résume très bien finalement... Merci pour toutes ces soirées « on regarde une série mais raconte-moi ce qu'il se passe dans ta vie en même temps ", pour les câlins de l'angoisse du jeudi soir, pour toutes ces bières au WAAW et ces discussions passionnantes que l'on peut avoir, même à $2 \mathrm{~g}$.

Clara, mon petit chou, de cointerne en enfer, on est devenue des amies, l'IHU nous a probablement bien rapprochée! Merci pour tout ce que tu m'as apporté sur ces 3 ans, de la bonne humeur, du kiffe, des rencontres, du love, merci pour les apéros sur ta terrasse 360 , pour ces soirées incroyables berthoms-sham-bunnys, les soirées aux chapiteaux, les après-midi loques à la plage le lendemain.

Mon Mathou, une rencontre formidable! Même si on ne se voit pas souvent, quand on se voit, on envoie du lourd! Merci pour ces soirées caipi au Chapiteau, cette soirée incroyable à faire les chœurs d'un SDF au cours Ju, je te prends au mot pour le semestre prochain, on va se le faire ce voyage!

\section{La rhumato,}

Emy, ma maman, qui m'a intégré à toute l'équipe à $\mathrm{J1}$, tu es la personne la plus douce, la plus gentille et la plus généreuse que j'ai rencontré, même s'il ne faut pas t'énerver pour autant ! Merci pour toutes ces soirées séries - thermomix, pour tous tes conseils rhumato et autres.

Caro, mon modèle, merci pour ton soutien, pour tous ce que tu m'as appris, tes bons conseils, pour tous ces détours pour me ramener chez moi après nos soirées rhumato, pour nous avoir intégré à ton voyage de thèse, qui ont été des vacances incroyables! J'espère qu'on pourra repartir en vacances ensemble !

Mon Juju, Tout a commencé quand tu m'as convaincu de rejoindre l'équipe de la rhumato à Marseille, merci pour ça déjà... Merci pour ton soutien sans faille, pour nos soirées pizza dark qui m’ont aidé à tenir le rythme cet été. Après l'Ecosse, l'Argentine, la Hongrie, on part ou après ?

Mon Lulu, comment est-on devenu amie à la base ? Aucune idée mais je sais que je ne pourrais plus me passer de toi maintenant ! Merci pour ta bienveillance, ton soutien inconditionnel, et ton humour surtout, j'ai hâte de rencontrer ton mini Toi. 
Clothilde, Merci d'avoir été ma 1ere assistante et de m'avoir appris la rhumatologie avec douceur et patience, tu es une personne et un médecin formidable!

Manu, un garçon incroyable, un super chef, le mec le plus populaire de sainte Marguerite, que dis-je de l'AP-HM ! Tu nous manques déjà beaucoup, j'espère que tu te plais à Lyon.

Dams, Douggy, Beubeu, puis Dodo, Justin, et Guillaume, la promo 2016 qui n'en finit plus de s'agrandir!

Dams, dams, merci d'avoir été un super cointerne, quoi 2 ou 3 fois ?

Mon Dodo, merci pour ta bonne humeur, quelle bonne idée d'avoir abandonné la gynéco pour la rhumato! On se refera vite des apéros aux akolytes ou au Ptit Pav promis!

Sonia, merci pour les soirées rhumato girls, et pour avoir été une super cointerne!

Théo, le baby du groupe, merci pour ces après-midis à écouter de la musique à l'autre bout du bureau, merci pour tes flatteries éhontées.

Lisa $\left(n^{\circ} 2\right)$, mon mini moi finalement, bouclée, souriante, et plus rigolote et sympa, je suis ravie que tu aies choisi rhumato à Marseille.

Lucas, Angélique, et Juliette, les rescapés de la promo 2017, je suis ravie que ce soit vous qui soyez resté, super relève de la rhumato.

Babs, Steph, Flo, merci pour tous ces apéros sur votre balcon er chez Emilie, pour l'expertise de Steph en rosé et la bonne humeur permanente de Flo. J'espère qu'on pourra très vite re faire des apéros et surtout retourner au Château!

L'équipe de neurologie,

Clémentine, Bull, Thomas, Faggia, Vincent, Bastien, Charline, un 1er semestre qui m'aura fait démarrer l'internat sur les chapeaux de roue, merci pour tous ces délires, pour m'avoir consolé quand on m'appelait la faucheuse.

Benji, le stage aura vraiment bien commencé, avec une super complicité et des bières sur la plage, j'en garde un souvenir incroyable, j'espère que toi aussi malgré tout, tu as été un cointerne de folie.

Nath et Marika, merci pour tout ce que vous m'avez appris, Marika, ça a été un plaisir de travailler avec toi, j'espère que tu te plairas à Montélimar ! 
Fanny, Françoise, Lulu et Eve, merci pour ces supers moments en hôpital de jour, pour m'avoir accepté puis supporté, même quand je n'étais pas physiquement présente mais que ma voix tournait en boucle dans l'HDJ (\#vidéo d'ETP de l'enfer)

Merci à audrey et Cathy en consultation pour leur aide pour recruter les patients.

Vous avez fait du super boulot, et finalement cette thèse ne se serait pas faite sans vous!

Mon Pitschou, je ne suis pas sûre que tu aies autant aimé ton stage que moi mais avoue, on aura bien rigolé ! Merci de m'avoir mise au vert, au no bra et 0 déchet ! Trop contente d'avoir pu garder contact avec toi et j'espère pouvoir bientôt venir te voir dans le Luberon!

Laveran

Professeur Thefenne, Arnaud, Thomas et Gratiane, merci pour vos enseignements, votre accueil incroyable et votre gentillesse, ce semestre a été très enrichissant et surtout très agréable !

Saly, Sonia, vous avez illuminé mon stage, merci pour votre bonne humeur et les chocolats chauds au gouter ;)

Merci à toutes les infirmières et kinés, vous faites un boulot incroyable, c'était un plaisir de travailler avec vous !

\section{Grenoble}

MC Solaar, le Tic de mon Tac, merci pour ta bonne humeur, tes gouts musicaux éclectiques (tu chercheras dans le dico;), merci pour les heures que tu as passé à essayer de faire mes stats, les vins chauds au marché de Noel, le ski pendant la grève, mon anniversaire confinement, nos paint magnifiques Marie, Charlotte, Océane, Grégoire et Caro, Dounia, merci pour le meilleur stage en confinement possible, pour toutes ces supers idées pour s'amuser malgré le COVID, retour de stage en rollers, corde à sauter et tours d'hôpital, les Pandemic Covid Movie Awards bien sûr! Vous m'avez fait kiffé mon interCHU !

Athan et Romain, merci pour ce semestre d'interCHU, merci de m'avoir si bien encadrée pour mon mémoire et merci pour votre bonne humeur et vos blagues incessantes.

Merci pour votre patience quand je ne comprenais rien à vos conversations statistiques... 
Laurent, le papa de l'HDJ qui m'avait déjà tout appris sur la rhumato en D4, merci pour ta bonne humeur, les visites en HDJ, " tous les feux sont au vert ! ", et tes poupounettes

\section{Kaplanski}

Meilleurs cointernes pour un dernier semestre, trop de kiffe, de love, d'Anticorps, de chansons et de nocturnes à la Conception.

JuJu Georgia, merci pour ta gentillesse, ta patience avec moi, tous les rides en scooter et tes invitations aux soirées colocs (hum hum cluster covid bonjour) JuJu Bermudez, l'autre " étranger "! Merci pour tout tes cours de pneumo et merci d'avoir fait semblant de t'intéresser à l'ostéoporose pour me faire plaisir...

Mon sergent colonel Bichon, merci pour ta gentillesse, pour les fous rires en t'entendant détruire des secrétaires/manip radio et autres au téléphone, sacré Bichon (à prononcer avec un énorme cheveu sur la langue)

Vincent $E$, merci d'avoir répondu à mes questions souvent débiles, avec gentillesse et sans jugement. Merci de m'avoir aidé à soumettre mon article pendant $1 \mathrm{~h}$ (blague j'avais oublié d'appuyer sur submit...) Thomas, merci pour ton humour, « docteur c'est grave si le cœur il bat plus...? »

Vincent $R, B O O O N$, merci pour tes explications et tes cours sur l'uvéite, merci pour tes expressions incrédules sous ton masque quand je dis des bêtises.

Karin, merci pour ta patience, ta rigueur et ta gentillesse, j'ai énormément appris dans ce stage, en grande partie grâce à toi.

Rodolphe, merci pour tes cours improvisés dans le bureau, tes blagues incroyables et tes imitations des tontons flingueurs avec GK.

$\mathrm{PA}$, merci pour ce que tu m'as appris, je t'enverrais tous mes syndromes de Jarrot, promis.

Je n'aurais pu rêver un meilleur stage et une meilleure équipe pour terminer en beauté mon internat. 


\section{TABLE DES MATIERES}

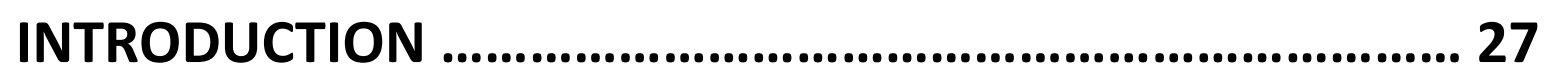

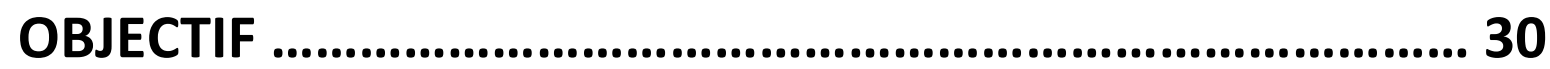

MATERIELS ET METHODE ............................................... 31

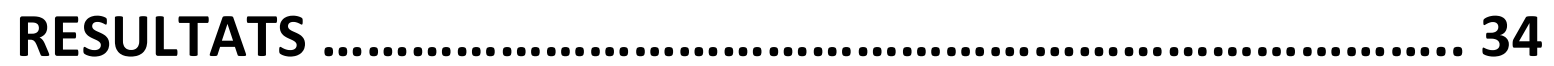

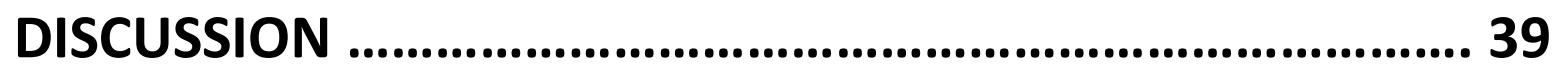

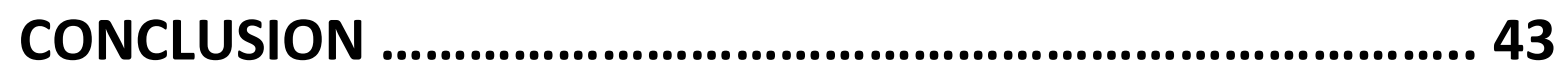

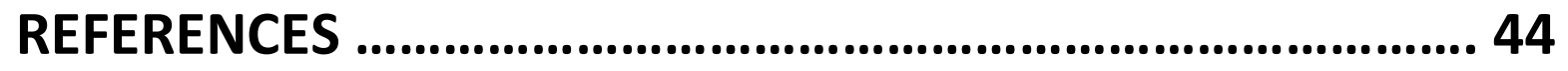

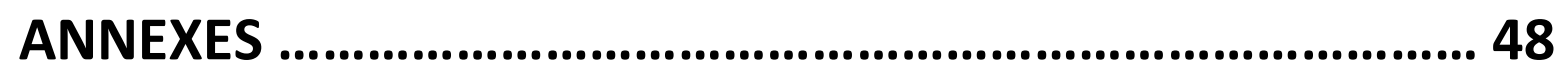

SERMENT D'HIPPOCRATE ................................................ 53

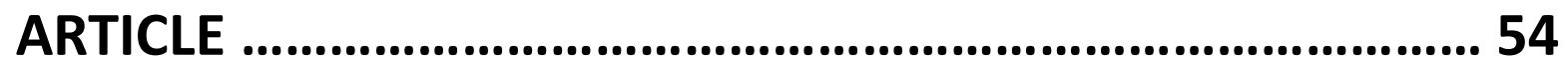




\section{INTRODUCTION}

Les rhumatismes inflammatoires regroupent plusieurs pathologies, les principaux étant: les spondyloarthrites dont font partie la spondylarthrite ankylosante (SA) (0,3 \% en France) (1) et le rhumatisme psoriasique $(0,12 \%)(2)$, et la polyarthrite rhumatoïde (PR) $(0,3-0,8 \%$ de la population française) (3). Ces pathologies atteignent préférentiellement les articulations et peuvent avoir un retentissement fonctionnel important altérant la qualité de vie des patients. La prise en charge de ces patients est complexe. Elle comprend en $1^{\text {ère }}$ intention des traitements médicamenteux, avec un arsenal thérapeutique de plus en plus large, notamment depuis l'arrivée des biothérapies [4-6].

Les progrès de cette prise en charge ont considérablement amélioré le pronostic fonctionnel et de la qualité de vie des patients. Cependant, les traitements actuels permettent d'atteindre une rémission ou une faible activité de la maladie chez environ 1 patient sur 2 seulement et nous ne sommes pas capables de guérir

maladie.

Les patients peuvent donc présenter de manière chronique des douleurs, des poussées inflammatoires, et même lorsque le rhumatisme est bien contrôlé, les patients rapportent des symptômes secondaires invalidants, tels que la fatigue chronique et un handicap fonctionnel persistant [12]. D'autre part, les traitements de fond, peuvent avoir des effets secondaires comme l'augmentation modérée du risque d'infection, des perturbations biologiques, ... Les possibles effets indésirables parfois surmédiatisés, le suivi clinique et paraclinique, peuvent être perçu comme dangereux ou contraignants, compromettant l'adhérence thérapeutique. 
Afin d'aider le patient dans la gestion de sa maladie, des ateliers d'échanges et d'information ciblés sur les besoins du patient, structurés sous l'appellation d'éducation thérapeutique (ETP), ont été développés. L'ETP est une pratique qui a toute sa place dans l'accompagnement des soins au patient. Elle est recommandée par les sociétés savantes de rhumatologie dans la prise en charge de ces pathologies [13-16]. Pour exemple, la Société Française de Rhumatologie recommande que «Tout patient atteint de PR devrait bénéficier d'une prise en charge globale incluant (...) des mesures d'éducation thérapeutique, ... » [17]

Le but de l'éducation thérapeutique est de permettre au patient d'être l'acteur principal de sa maladie, d'en obtenir une meilleure connaissance et d'acquérir des compétences d'auto-soins et d'adaptation.

En France, le déroulé du programme doit répondre à des directives précises établies par l'Agence Régionale de Santé (ARS) pour pouvoir mériter ce label : le programme se déroule en plusieurs étapes: le bilan éducatif partagé (BEP) permet d'évaluer les besoins spécifiques du patient, puis, un programme adapté composé de plusieurs ateliers permette d'aborder les sujets nécessaires et répondre aux questions posées lors du BEP. Enfin, lors d'un entretien final, les progrès réalisés et les attentes du patient sont réévalués et des ateliers complémentaires peuvent être proposés si nécessaires [18]

En pratique, l'éducation thérapeutique se sert de techniques de communication centrées sur le patient, ainsi que des techniques pédagogiques (exposés interactifs, des études de cas, des tables rondes, des simulations à partir de l'analyse d'une situation ou d'un carnet de surveillance, des travaux pratiques, atelier, simulations de gestes et de techniques, des activités sportives, des jeux 
de rôle, des témoignages documentaires), pour transmettre des compétences spécifiques au patient.

Tous les professionnels de santé peuvent participer à un programme d'éducation thérapeutique sous réserve d'avoir réalisé une formation à l'ETP.

Beaucoup d'études se sont intéressées à l'effet de l'ETP dans les maladies chroniques telles que le diabète, l'obésité, l'asthme avec des bénéfices à court et long terme sur la santé des patients [19]. Dans les rhumatismes inflammatoires, l'éducation thérapeutique a montré des bénéfices sur la diminution de la douleur, les connaissances sur la maladie, les comportements d'adaptation à court terme, le soutien social (20-23) ainsi que des effets à long terme sur l'activité de la maladie [24]. Il a également été montré des taux plus élevés d'adhésion après acquisition de connaissances sur des conventional synthetic Disease Modifying Anti Rheumatic Drugs (csDMARDS) lors d'un programme d'ETP [25]

Plus récemment, Fayet et al. ont mis en évidence que l'ETP permet une meilleure adhérence aux anti-Tumor Necrosis Factor (anti-TNF) et notamment lorsqu'elle était réalisée en séances individuelles [26].

Ainsi, l'éducation thérapeutique est un bon outil complémentaire pour permettre une prise en charge plus globale des patients atteints de rhumatisme inflammatoire.

Le bénéfice de l'éducation thérapeutique nécessite un intérêt, une démarche active du patient et une motivation à participer au programme et à ses ateliers. La majorité des travaux publiés a évalué l'efficacité des programmes d'ETP, mais peu ont évalué leur faisabilité. Or, comme énoncé en introduction, les programmes d'éducation thérapeutique comme définis par les ARS sont lourds en investissement pour les patients, qui doivent se libérer souvent pour plusieurs 
ateliers, parfois loin de leur domicile. Ces ateliers demandent également de I'investissement de la part des personnes, soignants ou non, qui les encadrent et les organisent. Il est donc primordial, pour une meilleure adhésion, de cibler au mieux les patients potentiellement intéressés.

A l'occasion de la mise en route à Marseille de 2 programmes d'éducation thérapeutique dédiés aux rhumatismes inflammatoires chroniques (Education dans les Rhumatismes Inflammatoires Chroniques ou ERIC et ETP RIC Réseau Santé Croisée), nous avons cherché à identifier les patients intéressés par ces programmes d'éducation et les facteurs associés à cet intérêt.

\section{OBJECTIF}

Notre objectif est d'identifier les patients atteints de rhumatismes inflammatoires chroniques intéressés par un programme d'éducation thérapeutique (ETP). 


\section{MATERIEL ET METHODES}

\section{1) Design de l'étude}

Il s'agissait d'un étude prospective observationnelle (non-interventionnelle), incluant les patients consécutifs entre septembre et octobre 2019.

Nous avons recueilli les données des patients vus pour un rhumatisme inflammatoire chronique au CHU de Marseille (hôpital Sainte Marguerite, APHM) en hôpital de jour et en consultation sur une durée de quatre semaines.

Cette étude utilisant les données de suivi des patients recueillies de façon habituelle dans le cadre du soin (lors de la proposition du programme d'ETP), il ne s'agit donc pas d'une RIPH (Recherche Impliquant la Personne Humaine) et ne nécessite donc pas la saisie d'un Comité de Protection des Personnes (CPP). La demande d'accès aux données a été présentée au PADS de l'APHM selon la procédure interne prévue.

2) Population:

Les critères d'inclusion comprenaient un âge supérieur à 18 ans, un diagnostic de rhumatisme inflammatoire chronique ( $S p A$, RhPso ou PR) par un rhumatologue.

\section{3) Méthodologie}

Les patients étaient informés systématiquement au cours de leur venue en hôpital de jour ou en consultation de la mise en place d'un programme d'éducation thérapeutique. Il leur était alors proposé de visionner une vidéo d'explication enregistrée dans le service sur le principe de l'éducation thérapeutique (annexe 1, lien vidéo: https://youtu.be/jw5eJejSDXE). Les 
patients étaient ensuite invités à répondre à un questionnaire (annexe 2), après avoir obtenu leur accord.

Des brochures comportant des informations pratiques concernant les deux programmes d'éducation thérapeutique disponibles sur Marseille leur étaient également remises en même temps que le questionnaire par l'équipe soignante de la consultation ou de l'hôpital de jour.

\section{4) Modalités de recueil des données}

Les données concernant le patient et la maladie étaient collectées sur le dossier médical informatisé (DMI) : âge, sexe, profession, les critères diagnostiques définis selon American College of Rheumatology-EULAR pour la PR, I'Assessment of SpondyloArthritis International Society (ASAS) pour les SpA, l'activité de la maladie selon le Disease Activity Score 28 et le Bath Ankylosing Spondylitis Disease Activity Index (BASDAI) recueillis dans les compte-rendus de consultation et d'hôpital de jour, les effets indésirables des biothérapies dans les 12 mois qui précédaient l'évaluation. Les rhumatismes inflammatoires des patients étaient considérés comme actifs pour la SpA si le BASDAI était $>4$, pour la PR si le DAS 28 était strictement supérieur à 3,2 et pour le rhumatisme psoriasique, le BASDAI ou le DAS 28 étaient utilisés, ou à l'appréciation du rhumatologue si ces données n'étaient pas disponibles. L'ancienneté du diagnostic était recueillie à la fois dans les questionnaires et sur le DMI. Les données psycho-sociales telles que la catégorie socioprofessionnelle selon la classification INSEE (annexe 3), le statut professionnel (en activité, arrêt, invalidité...), ainsi que l'intérêt porté à l'ETP après information étaient recueillis via le questionnaire. 
Le questionnaire posait également des questions qualitatives afin d'explorer les raisons de l'intérêt ou non pour le programme d'ETP et les obstacles envisagés par le patient pour y participer (Annexe 2)

\section{5) Critère de jugement principal}

Notre critère de jugement principal était le pourcentage de patient montrant un intérêt pour le programme d'éducation thérapeutique, information apportée par le questionnaire initial à la question «seriez-vous intéressés par un programme d'ETP ».

\section{6) Analyses statistiques}

Les variables d'intérêt ont été présentées sous forme de fréquence absolue et relative (\%) selon les deux groupes d'intérêt ou non pour l'ETP. Pour comparer les différences entre le groupe intéressé par l'ETP et le groupe non intéressé, nous avons utilisé le test du Chi2, pour toutes les variables d'intérêt qualitatives (ou test de Fisher lorsque les conditions d'application du Chi2 n'étaient pas réunies).

Un modèle de régression logistique pour l'analyse multivariée, incluant les variables significatives au seuil 0,2 en analyse univariée, a ensuite été utilisé pour évaluer l'effet propre de chacun de ces facteurs (indépendamment des autres facteurs) sur l'intérêt pour l'ETP. L'odds ratio correspondant à ces associations a été calculé en univariée et en multivarié (ainsi que son intervalle de confiance à $95 \%$ pour l'analyse multivarié). Aucune imputation pour les données manquantes n'a été appliquée.

Le seuil alpha a été fixé à 0,05 (sous hypothèse alternative bilatérale) pour tous les tests, sans correction pour la multiplicité des tests compte tenu du caractère exploratoire de cette étude. 
Le logiciel IBM ${ }^{\circledR}$ SPSS $^{\circledR}$ Statistics version 20.0 .0 a été utilisé pour l'analyse.

\section{RESULTATS}

Caractéristiques de la population

Au total, 194 patients ont été inclus entre septembre et octobre 2019. Parmi eux, 184 patients étaient éligibles. Un total de 183 patients a été inclus pour les analyses ( 1 refus de participer à l'étude). La sélection des patients est rapportée sur la figure 1. 


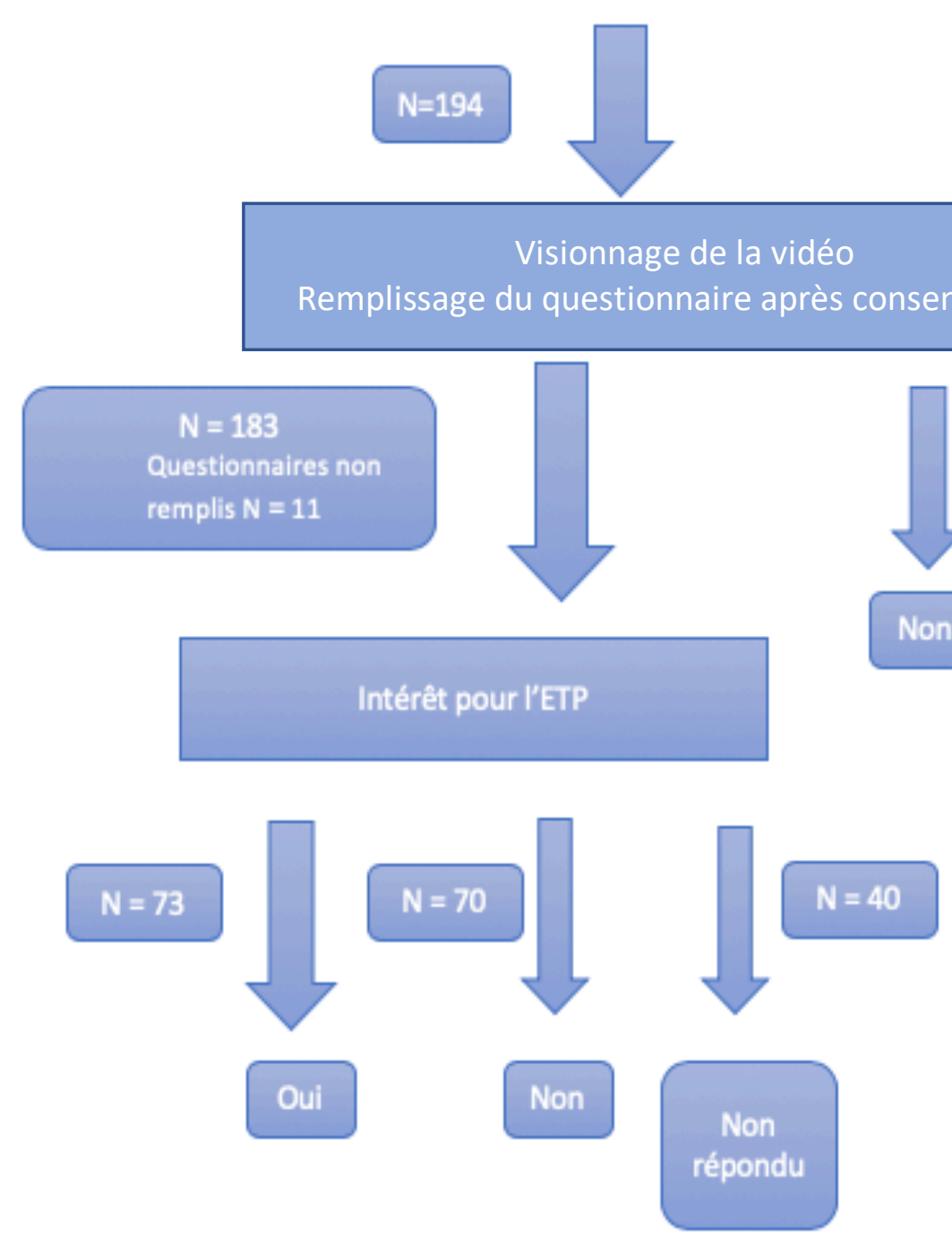

Figure 1 : Flowchart et déroulement de l'étude

A l'inclusion, il y avait 126 femmes (69 \%) pour 57 hommes (31 \%) d'âge moyen de 53,2 ans.

Les pathologies étaient 86 spondyloarthrites (48\%), 55 polyarthrites rhumatoïdes (30\%) et 40 rhumatismes psoriasiques (22\%). Cent dix-huit (64 \%) patients étaient en rémission contre 41 (22\%) en activité. 
Cent quarante-quatre patients $(79 \%)$ étaient traités par biothérapies au moment de l'inclusion, 33 étaient traités par csDMARDS seuls. 85 patients ont été recrutés en hôpital de jour et 98 en consultation.

\section{Facteurs associés à l'intérêt pour l'ETP en univariée}

Concernant le critère de jugement principal, sur les 183 patients, la donnée était disponible dans 143 questionnaires (78,1\%). Parmi ceux-ci, 70 n'étaient pas intéressés (49 \%) contre 73 intéressés par l'ETP (51\%).

En analyse univariée, (tableau 1) un diagnostic récent était significativement associé avec un intérêt à l'ETP $(O R=5,46 ; p=0,024)$. Le lieu d'évaluation influençait également l'intérêt : le fait d'être évalué en consultation plutôt qu'en hôpital de jour était significativement associé à un plus grand intérêt à l'ETP $(\mathrm{OR}=\mathbf{7 , 5 6} ; \mathrm{p}=0,007)$.

Un âge plus jeune était également associé de manière significative à un intérêt pour l'ETP $(O R=2 ; p=0,046)$.

Le sexe, le traitement par biothérapie, l'incidence d'évènements indésirables dans les 12 mois ainsi que l'activité de la maladie n'était pas statistiquement associée avec l'intérêt pour l'éducation thérapeutique.

A propos des catégories socio-professionnelles, les catégories 4 et 5 semblaient surreprésentées dans le groupe d'intérêt pour l'ETP. Ces catégories regroupent les professions intermédiaires (exemple parmi nos patients : professeurs des écoles, préparateur en pharmacie, infirmière...) pour la catégorie 4 et les employés pour la catégorie 5 (fonctionnaire, aide-soignante, policier...). A l'inverse, les patients sans emploi et dans la catégorie 6 (ouvriers) paraissaient surreprésentés dans le groupe non intéressé par l'ETP.

Inversement, les patients plus âgés, recrutés en HDJ étaient associés à une absence d'intérêt pour l'ETP. 
Tableau 1 : Caractéristiques des patients en fonction de leur intérêt pour l'ETP

\begin{tabular}{|c|c|c|c|c|c|}
\hline & & \multirow{2}{*}{\multicolumn{2}{|c|}{ Intérêt ETP }} & \multirow{3}{*}{$\mathrm{OR}^{*}$} & \multirow{3}{*}{$p$} \\
\hline & & & & & \\
\hline & & Non $(n=70)$ & Oui $(n=73)$ & & \\
\hline \multirow[t]{3}{*}{ Pathologie } & SPA n (\%) & $31(47,7)$ & $34(52,3)$ & 2,6 & 0,274 \\
\hline & $P R, n(\%)$ & $25(58,1)$ & $18(41,9)$ & & - \\
\hline & Rh Pso, n (\%) & $14(40,0)$ & $21(60,0)$ & & - \\
\hline \multirow{2}{*}{$\begin{array}{l}\text { Date du } \\
\text { diagnostic (an) }\end{array}$} & $<5, \mathrm{n}(\%)$ & $9(30,0)$ & $21(70,0)$ & 5,5 & 0,024 \\
\hline & $>5 \mathrm{n}(\%)$ & $61(54,0)$ & $52(46,0)$ & & - \\
\hline \multirow[t]{2}{*}{$\begin{array}{l}\text { Activité } \\
\text { maladie }\end{array}$} & $\begin{array}{l}\text { Rémission/faible, } n \\
\text { (\%) }\end{array}$ & $47(52,8)$ & $42(47,2)$ & 2,5 & 0,162 \\
\hline & Forte, n (\%) & $13(37,1)$ & $22(62,9)$ & & \\
\hline \multirow[t]{2}{*}{ Biothérapie } & Oui n (\%) & $59(51,3)$ & $56(48,7)$ & 0,4 & 0,660 \\
\hline & Non n (\%) & $11(44,0)$ & $14(56,0)$ & & \\
\hline \multirow{2}{*}{$\begin{array}{ll}\text { Lieu } & \text { de } \\
\text { proposition } & \end{array}$} & HDJ, n (\%) & $39(61,9)$ & $24(38,1)$ & 7,6 & 0,007 \\
\hline & Consultation, n (\%) & $31(38,8)$ & $49(61,3)$ & & \\
\hline \multirow[t]{2}{*}{ Sexe } & Homme, n (\%) & $24(53,3)$ & $21(46,7)$ & 0,5 & 0,589 \\
\hline & Femme, n (\%) & $46(49,9)$ & $52(53,1)$ & & \\
\hline Age, an (DS) & & $54.6(14,5)$ & $49,9(12,7)$ & 2 & 0,046 \\
\hline \multirow{3}{*}{$\begin{array}{l}\text { Niveau } \\
\text { d'éducation }\end{array}$} & Brevet & $18(45,0)$ & $22(55,0)$ & 1,4 & 0,489 \\
\hline & $\mathrm{Bac}$ & $24(53,3)$ & $21(46,7)$ & & \\
\hline & Supérieur & $18(40,9)$ & $26(59,1)$ & & \\
\hline \multirow{2}{*}{$\begin{array}{l}\text { Langue } \\
\text { maternelle }\end{array}$} & Française & $61(49,2)$ & $63(50,8)$ & 0,03 & 1 \\
\hline & Autres & $7(46,7)$ & $8(53,3)$ & & \\
\hline En activité & Oui & $29(42,6)$ & $39(57,4)$ & 1,6 & 0,135 \\
\hline \multirow{7}{*}{$\begin{array}{l}\text { Catégories socio } \\
\text { professionnelles }\end{array}$} & Sans & $8(72,7)$ & $3(27,3)$ & 15,3 & 0,015 \\
\hline & 1 & $19(59,4)$ & $13(40,6)$ & - & - \\
\hline & 2 & $3(50,0)$ & $3(50,0)$ & - & - \\
\hline & 3 & $15(45,5)$ & $18(54,5)$ & - & - \\
\hline & 4 & $7(35,0)$ & $13(65,0)$ & - & - \\
\hline & 5 & $10(31,3)$ & $22(68,8)$ & - & - \\
\hline & 6 & $8(88,9)$ & $1(11,1)$ & - & - \\
\hline $\begin{array}{l}\text { Effets } \\
\text { indésirables }<12 \\
\text { mois }\end{array}$ & Oui & $25(53,2)$ & $22(46,8)$ & 0,4 & 0,593 \\
\hline
\end{tabular}

* test du chi2 pour toutes les variables qualitatives vs intérêt (ligne 1 à 9 du tableau), test de Fisher (effectifs trop petits) pour catégorie socio professionnelle, test t de student pour l'âge. 
Facteurs indépendamment associés à un intérêt pour l'ETP en analyse multivariée

Une régression logistique pour évaluer le poids de chacune des variables indépendamment des autres a ensuite été réalisée (toutes les variables au seuil significatif de 0,2 ont été intégrées y compris des variables non significatives au seuil classique de 0,05 c'est-à-dire : l'âge, la date de diagnostic, l'activité de la maladie, l'activité professionnelle, le lieu de proposition) pour aboutir au modèle final.

Seuls l'ancienneté du rhumatisme inflammatoire et le lieu de proposition apparaissent dans le modèle final, avec un résultat statistiquement significatif pour le lieu d'évaluation. L'évaluation en consultation est donc indépendamment associée à l'intérêt pour l'ETP $(O R=2,3(1,1 ; 4.8) ; p=0,031)$

Tableau 2 : Analyse de régression logistique multivariée des facteurs associés à l'intérêt à l'éducation thérapeutique.

\begin{tabular}{|c|c|c|c|c|}
\hline & $\begin{array}{l}\mathrm{OR} \\
\text { univarié }\end{array}$ & P value & OR multivarié (IC $95 \%$ ) & $P$ value \\
\hline Ancienneté du diagnostic & 5,5 & 0,024 & $0 ., 03(0,15 ; 1,1)$ & 0,071 \\
\hline $\begin{array}{l}\text { Lieu de proposition } \\
\text { Consultation vs HDJ }\end{array}$ & 7,6 & 0,007 & $2,3(1,1 ; 4,8)$ & 0,031 \\
\hline $\begin{array}{l}\text { En activité } \\
\text { (professionnelle) }\end{array}$ & 1,6 & 0,13 & $1,5(0,7 ; 3,2)$ & 0,311 \\
\hline Activité de la maladie & 2,5 & 0,16 & $1,5(0,7 ; 3,6)$ & 0,311 \\
\hline
\end{tabular}




\section{Résultats qualitatifs}

D'autre part, dans le questionnaire, nous avons également inclus des questions ouvertes pour explorer les raisons de l'intérêt ou non à l'éducation thérapeutique.

Concernant l'absence d'intérêt, les patients abordaient principalement la localisation trop éloignée de leur domicile (28 patients sur 184).

Certains patients ont évoqué une autonomie et une gestion de la maladie déjà acquises, plutôt parmi les patients recrutés en consultation 8,2 \% (8/98) qu'en hôpital de jour 3,5 \% (3/85)

Le besoin de connaissances sur la maladie, meilleure gestion de la maladie au quotidien, l'amélioration de la qualité de vie ainsi que le soutien psychologique et social étaient les éléments principaux suggérés par les patients intéressés par le programme d'ETP.

\section{DISCUSSION}

Cette évaluation prospective avait pour but d'identifier au mieux les patients intéressés par l'ETP. Nous avons retrouvé que la moitié des patients étaient intéressés, et que les patients plus jeunes, ayant un diagnostic plus récent ainsi que les patients suivis préférentiellement en consultation sont ceux qui sont les plus intéressés. C'est la première étude, à notre connaissance, qui évalue en France et dans les rhumatismes inflammatoires, l'intérêt des patients pour un programme d'ETP, permettant ainsi de mieux cibler les patients lors de la proposition initiale d'un programme d'ETP. 
En effet les besoins éducationnels des patients ont été évalués à plusieurs reprises, mais peu d'études rapportent le profil de patients en demande d'information. Ainsi, l'équipe française de Perdriger, référente en ETP, a fait une large mise au point sur les besoins éducationnels des rhumatismes inflammatoires pour permettre de mieux cibler les attentes des patients [27]. Néanmoins, elle ne s'intéresse qu'au contenu de l'information transmise et ne permet pas de connaître les profils des patients en demande d'information. D’autres études ont évalué ce paramètre et confirment que le diagnostic récent et l'âge jeune sont associés à un besoin éducationnel plus important $[28,29]$.

Ces résultats semblent confirmer notre pratique clinique. En effet, le moment du diagnostic est un moment clé où le patient nécessite des informations claires sur sa pathologie, les traitements et complications de ces traitements.

Nous retrouvons dans notre étude, $50 \%$ environ de patients non intéressés par I'ETP, notamment les patients dont le diagnostic est ancien et sont suivis très régulièrement en hôpital de jour. En effet, certains patients ne ressentent pas de besoin d'information, et gèrent leur maladie au quotidien depuis de nombreuses années.

L'intérêt pour l'ETP est retrouvé chez la moitié des rhumatismes inflammatoires chroniques qui doivent donc être identifiés afin de pouvoir leur proposer une éducation adaptée.

II est également possible que les patients ne soient pas intéressés par le programme proposé et non par l'ETP en général, en raison de la localisation des programmes notamment, comme suggérés dans les réponses qualitatives, évoquées dans les résultats. 
Une proportion non négligeable de patients (21\%) n'a pas répondu à la question principale sur l'intérêt pour l'ETP. II est possible que ce soit par manque de compréhension et que la présentation que nous leur avons faite de l'ETP n'ait pas été suffisamment claire. En effet l'ETP peut être un concept difficile à appréhender, et elle est rendue compliquée par la législation qui l'entoure et lui donne un cadre rigide.

La capacité des patients à comprendre et traiter des informations liées à la santé afin de prendre des décisions appropriées sur leur santé [30] peut être un obstacle éducationnel et culturel à l'ETP [31]. Ce concept est étudié sous le nom de " health literacy " et n'est pas rare : environ $10 \%$ des patients atteints de polyarthrite rhumatoïde n'auraient pas les outils nécessaires pour comprendre des consignes basiques concernant leur traitement et leur suivi [31]. Ces patients, moins à l'aise avec leur maladie, bénéficieraient probablement de I'ETP, d'autant qu'un bas niveau de " health literacy " est corrélé au handicap fonctionnel dans la polyarthrite rhumatoïde [32].

Une meilleure communication dans la présentation de l'ETP est donc nécessaire afin de la rendre plus accessible et plus compréhensible.

Par ailleurs, nous n'avons pas pu montrer que les patients ayant un rhumatisme inflammatoire actif ni que les patients ayant présenté des effets indésirables liés aux biothérapies étaient plus intéressés par l'ETP. Pourtant ces facteurs sont rapportés de manière intuitive par les professionnels pratiquant l'ETP et sont décrits dans la littérature [33]. En effet, ce sont des situations de fragilité dans lesquelles le patient a le plus besoin de support et donc potentiellement d'ETP. Il est possible que nous ayons rencontré un manque de puissance qui ne nous permette pas de mettre en évidence l'influence de ces 2 paramètres: nous avions peu de patients en activité forte de leur maladie ainsi que peu 
d'occurrences d'évènements indésirables dans notre cohorte.

Concernant le niveau d'éducation, nous n'avons pas retrouvé de différence d'intérêt selon le niveau d'étude. II est retrouvé dans la littérature que les patients avec un niveau d'éducation plus faible étaient plus susceptibles d'améliorer leurs connaissances grâce à I'ETP [34]. Cependant, nous retrouvons dans notre étude, une tendance à un intérêt plus important pour les catégories socioprofessionnelles plus élevées, possiblement en rapport avec un aspect scolaire de l'ETP dans sa forme actuelle.

Notre étude monocentrique a certaines limites. Les rhumatismes inflammatoires sont en grande partie suivis en médecine de ville, or nos patients ont été évalués au CHU uniquement. Cependant nous avons axé sur les patients ambulatoires en hôpital de jour et en consultations pour pouvoir être le plus représentatif.

Par ailleurs, il est fort probable que la manière de présenter et de proposer l'éducation thérapeutique selon la connaissance qu'en a chaque médecin ou soignant diffère beaucoup. C'est pour limiter l'impact de ce paramètre et uniformiser au maximum l'information reçue sur l'éducation thérapeutique que nous avons créé une vidéo explicative. Cependant ce choix a aussi des limites, en effet nous n'avons pu évaluer l'impact qu'aurait eu l'information par le praticien traitant, or l'information est mieux accueillie lorsqu'elle est délivrée par celui-ci plutôt qu'un intervenant non connu du patient.

La deuxième limite de notre étude est liée au contexte sanitaire. Nous aurions souhaité évaluer la proportion de patients débutant les ateliers d'ETP. Nous pressentions que sur les $50 \%$ intéressés, seule une faible proportion de patients aurait réalisé la démarche active d'initier le programme et le terminer, ce qui est une donnée non connue dans la littérature. 
Malheureusement, nous n'avons pu l'obtenir en raison de la survenue de l'épidémie de COVID-19 qui a suspendu les différents programmes d'ETP de Marseille pendant plusieurs mois. Nous espérons pouvoir poursuivre ces investigations lorsque le contexte sanitaire le permettra.

\section{CONCLUSION}

Dans notre étude prospective monocentrique, nous avons montré que certains critères tels qu'un diagnostic récent et une proposition individuelle en consultation étaient prédictifs d'un intérêt plus important pour les programmes d'éducation thérapeutique.

L'éducation thérapeutique est un outil complémentaire cruciale dans la prise en charge du patient, mais pour être utile, elle doit être proposée au bon moment et au bon patient. D'autres études sont nécessaires pour confirmer ces données, mais il semblerait que les patients jeunes au début de leur prise en charge seraient des critères intérêt à une prise en charge en éducation thérapeutique. Un travail sur la communication autour de l'ETP pour les soignants et les patients doit être envisagé pour pouvoir rendre cette pratique plus compréhensible et accessible au plus grand nombre. 


\section{REFERENCES}

1. Site ameli, spondylarthrite ankylosante [Internet]. Disponible sur: https://www.ameli.fr/assure/sante/themes/spondylarthriteankylosante/definition-facteurs-favorisants

2. Cofer, rhumatisme psoriasique [Internet]. Disponible sur: http://www.lecofer.org/item-cours-1-7.php

3. Site ameli, polyarthrite rhumatoide [Internet]. Disponible sur: https://www.ameli.fr/assure/sante/themes/polyarthrite-rhumatoide/comprendrepolyarthrite-rhumatoide

4. Gossec L, Smolen JS, Ramiro S, de Wit M, Cutolo M, Dougados M, et al. European League Against Rheumatism (EULAR) recommendations for the management of psoriatic arthritis with pharmacological therapies: 2015 update. Ann Rheum Dis. mars 2016;75(3):499-510.

5. Smolen JS, Landewé R, Breedveld FC, Buch M, Burmester G, Dougados $\mathrm{M}$, et al. EULAR recommendations for the management of rheumatoid arthritis with synthetic and biological disease-modifying antirheumatic drugs: 2013 update. Ann Rheum Dis. mars 2014;73(3):492-509.

6. van der Heijde D, Ramiro S, Landewé R, Baraliakos X, Van den Bosch F, Sepriano A, et al. 2016 update of the ASAS-EULAR management recommendations for axial spondyloarthritis. Ann Rheum Dis. juin 2017;76(6):978-91.

7. Allaart CF, Goekoop-Ruiterman YPM, de Vries-Bouwstra JK, Breedveld FC, Dijkmans B a. C, FARR study group. Aiming at low disease activity in rheumatoid arthritis with initial combination therapy or initial monotherapy strategies: the BeSt study. Clin Exp Rheumatol. déc 2006;24(6 Suppl 43):S-7782.

8. Goekoop-Ruiterman YPM, De Vries-Bouwstra JK, Allaart CF, Van Zeben D, Kerstens PJSM, Hazes JMW, et al. Clinical and radiographic outcomes of four different treatment strategies in patients with early rheumatoid arthritis (the BeSt study): A randomized, controlled trial. Arthritis Rheum. nov 2005;52(11):3381-90.

9. Mease PJ, Smolen JS, Behrens F, Nash P, Liu Leage S, Li L, et al. A head-to-head comparison of the efficacy and safety of ixekizumab and adalimumab in biological-naïve patients with active psoriatic arthritis: 24-week results of a randomised, open-label, blinded-assessor trial. Ann Rheum Dis. janv 2020;79(1):123-31.

10. Marzo-Ortega H, Sieper J, Kivitz A, Blanco R, Cohen M, Martin R, et al. Secukinumab and Sustained Improvement in Signs and Symptoms of Patients With Active Ankylosing Spondylitis Through Two Years: Results From a Phase III Study: Two-Year Efficacy and Safety Results in AS With Secukinumab. Arthritis Care Res. juill 2017;69(7):1020-9. 
11. Maksymowych WP, Dougados M, van der Heijde D, Sieper J, Braun J, Citera G, et al. Clinical and MRI responses to etanercept in early nonradiographic axial spondyloarthritis: 48-week results from the EMBARK study. Ann Rheum Dis. juill 2016;75(7):1328-35.

12. Taylor PC, Moore A, Vasilescu R, Alvir J, Tarallo M. A structured literature review of the burden of illness and unmet needs in patients with rheumatoid arthritis: a current perspective. Rheumatol Int. mai 2016;36(5):685-95.

13. Zochling J. ASAS/EULAR recommendations for the management of ankylosing spondylitis. Ann Rheum Dis. 1 avr 2006;65(4):442-52.

14. Zangi HA, Ndosi M, Adams J, Andersen L, Bode C, Boström C, et al. EULAR recommendations for patient education for people with inflammatory arthritis. Ann Rheum Dis. juin 2015;74(6):954-62.

15. Combe B, Landewe R, Lukas C, Bolosiu HD, Breedveld F, Dougados M, et al. EULAR recommendations for the management of early arthritis: report of a task force of the European Standing Committee for International Clinical Studies Including Therapeutics (ESCISIT). Ann Rheum Dis. 30 juin 2006;66(1):34-45.

16. Deighton C, O'Mahony R, Tosh J, Turner C, Rudolf M, on behalf of the Guideline Development Group. Management of rheumatoid arthritis: summary of NICE guidance. BMJ. 16 mars 2009;338(mar16 1):b702-b702.

17. Daien C, Hua C, Gaujoux-Viala C, Cantagrel A, Dubremetz M, Dougados $\mathrm{M}$, et al. Actualisation des Recommandations de la Société française de rhumatologie pour la prise en charge de la polyarthrite rhumatoïde. Rev Rhum. janv 2019;86(1):8-24.

18. Site HAS, Education thérapeutique [Internet]. Disponible sur:

https://www.has-sante.fr/jcms/r_1496895/fr/education-therapeutique-du-patientetp

19. Newman S, Steed L, Mulligan K. Self-management interventions for chronic illness. The Lancet. oct 2004;364(9444):1523-37.

20. Stenberg U, Haaland-Øverby M, Fredriksen K, Westermann KF, Kvisvik $\mathrm{T}$. A scoping review of the literature on benefits and challenges of participating in patient education programs aimed at promoting self-management for people living with chronic illness. Patient Educ Couns. nov 2016;99(11):1759-71. 21. Røe R, Grønning K, Eriksson LR, Zangi HA. Outcomes in patient education programmes for people with rheumatic diseases: Results from a Delphi process and a study of feasibility and responsiveness. Musculoskeletal Care. 28 janv 2020;msc. 1456.

22. Riemsma RP, Taal E, Kirwan JR, Rasker JJ. Systematic review of rheumatoid arthritis patient education: RA Patient Education: Review. Arthritis Care Res. 15 déc 2004;51(6):1045-59.

23. Niedermann K, Fransen J, Knols R, Uebelhart D. Gap between short- and long-term effects of patient education in rheumatoid arthritis patients: A 
systematic review: Patient Education in RA Patients. Arthritis Care Res. 15 juin 2004;51(3):388-98.

24. Abourazzak F, El Mansouri L, Huchet D, Lozac'hmeur R, HajjajHassouni N, Ingels A, et al. Long-term effects of therapeutic education for patients with rheumatoid arthritis. Joint Bone Spine. déc 2009;76(6):648-53.

25. Hill J, Bird H, Johnson S. Effect of patient education on adherence to drug treatment for rheumatoid arthritis: a randomised controlled trial. Ann Rheum Dis. sept 2001;60(9):869-75.

26. Fayet F, Fan A, Rodere M, Savel C, Pereira B, Soubrier M. Adherence to Subcutaneous Anti-TNF Treatment in Chronic Inflammatory Rheumatism and Therapeutic Patient Education. Patient Prefer Adherence. févr 2020;Volume 14:363-9.

27. Perdriger A, Pouplin S, Dartois A, Beauvais C. Objectifs et besoins éducatifs des patients atteints de rhumatismes inflammatoires chroniques. Rev Rhum Monogr. juin 2013;80(3):152-6.

28. Meesters JJL, Vliet Vlieland TPM, Hill J, Ndosi ME. Measuring educational needs among patients with rheumatoid arthritis using the Dutch version of the Educational Needs Assessment Tool (DENAT). Clin Rheumatol. sept 2009;28(9):1073-7.

29. Marques ML, Ferreira RJO, Machado PM, Marques A, da Silva JAP, Ndosi M. Educational needs in people with ankylosing spondylitis and psoriatic arthritis: a cross-sectional study. Clin Exp Rheumatol. avr 2020;38(2):282-8.

30. CDC. Health Litteracy [Internet]. Disponible sur: https://www.cdc.gov/healthliteracy/learn/index.html

31. Buchbinder R, Hall S, Youd JM. Functional health literacy of patients with rheumatoid arthritis attending a community-based rheumatology practice. J Rheumatol. mai 2006;33(5):879-86.

32. Caplan L, Wolfe F, Michaud K, Quinzanos I, Hirsh JM. Strong Association of Health Literacy With Functional Status Among Rheumatoid Arthritis Patients: A Cross-Sectional Study: Health Literacy and RA Functional Status. Arthritis Care Res. avr 2014;66(4):508-14.

33. Bremander A, Haglund E, Bergman S, Ndosi M. The educational needs of patients with undifferentiated spondyloarthritis: Validation of the ENAT questionnaire and needs assessment. Musculoskeletal Care. juin 2018;16(2):313-7.

34. Lopez-Olivo MA, Lin H, Rizvi T, Barbo A, Ingleshwar A, des Bordes JKA, et al. Randomized Controlled Trial of Patient Education Tools for Patients with Rheumatoid Arthritis. Arthritis Care Res. 25 juin 2020;acr.24362.

35. Combe B, Landewe R, Daien CI, Hua C, Aletaha D, Álvaro-Gracia JM, et al. 2016 update of the EULAR recommendations for the management of early arthritis. Ann Rheum Dis. juin 2017;76(6):948-59. 


\section{ANNEXES}

\section{Annexe 1 : Discours type lu dans la vidéo}

"Dans le cadre de la prise en charge de votre rhumatisme inflammatoire, nous souhaiterions vous proposer de participer à un programme d'éducation thérapeutique.

De manière générale, l'objectif de l'éducation thérapeutique est de vous permettre de mieux vivre avec votre maladie.

Au travers d'ateliers interactifs répartis sur l'année, nous vous proposons d'acquérir des connaissances sur votre rhumatisme, votre traitement, trouver des solutions pour diminuer vos douleurs et rester actif... Tout cela afin de vous permettre de mieux gérer votre maladie au quotidien et de manière autonome.

Comment ça se déroule?

Tout d'abord une $1^{\text {ère }}$ consultation pour mieux vous connaître et cibler vos besoins et vos envies spécifiques d'apprentissage.

En fonction de ces besoins, nous vous proposerons des ateliers adaptés (entre 2 et 3).

Enfin, nous réaliserons ensemble un bilan final nous permettant d'évaluer les progrès réalisés.

Ce programme est gratuit et s'intègre dans la prise en charge de votre rhumatisme inflammatoire pour améliorer votre qualité de vie ».

Lien vidéo Youtube : https://youtu.be/jw5eJejSDXE 
Annexe 2 : Questionnaire de recueils de données : L'éducation thérapeutique dans les rhumatismes

- Nom:

- Prénom :

- Date de naissance :

- Sexe : Homme / Femme

- Niveau d'étude : Brevet / baccalauréat / études supérieures

- Profession :

○ En activité 
- Temps plein :

- retraité :

- Arrêt de travail

- Invalidité

- Quelle est votre maladie (Rhumatisme psoriasique, polyarthrite rhumatoïde, spondylarthrite ankylosante) :

- Depuis combien de temps avez-vous cette maladie ?

$-\quad<3$ mois

- $\quad 3-6$ mois

- 6 - 12 mois

$-1-5$ ans

$->5$ ans

- Qui réalise votre suivi ?

- Mon médecin de famille

- Mon rhumatologue de ville

- Mon rhumatologue hospitalier

- Vers qui vous tournez-vous en cas de question ? (Médecin traitant, famille, amis, association, internet...) : 
- Comment évaluez-vous l'activité de votre maladie entre 0 et 10 :

- Êtes-vous satisfait(e) de votre prise en charge, note entre 0 et 10 :

- Qu'est ce qui pourrait être amélioré ?

- Seriez-vous intéressé par le programme d'éducation thérapeutique ? Si oui ou non, pourquoi?

- Quelles seraient vos disponibilités pour l'éducation thérapeutique ? (matin, après midi, soir...)

Merci de votre participation!» 


\section{Annexe 3 : classification INSEE}

\begin{tabular}{|c|c|c|c|c|}
\hline & \multicolumn{2}{|c|}{ INDIVIDUS } & \multicolumn{2}{|c|}{$\begin{array}{l}\text { MÉNAGES } \\
\text { classés selon la CS du chef }\end{array}$} \\
\hline$C S P$ & $\begin{array}{c}\text { Nombre } \\
\text { (en milliers) }\end{array}$ & $\begin{array}{c}\% \text { de la } \\
\text { population } \\
\text { active }\end{array}$ & $\begin{array}{l}\text { Nombre } \\
\text { (en milliers) }\end{array}$ & $\begin{array}{l}\% 0 \text { du total } \\
\text { des ménages } 1\end{array}$ \\
\hline $\begin{array}{l}\text { 0. AGRICULTEURS EXPLOITANTS } \ldots \ldots \ldots \ldots \ldots \ldots \\
\text { 00. Agriculteurs exploitants } \ldots \ldots \ldots \ldots \ldots\end{array}$ & 1448 & 62 & 800 & 41 \\
\hline $\begin{array}{l}\text { 1. SALARIÉS AGRIColes } \ldots \ldots \ldots \ldots \ldots \ldots \ldots \ldots \ldots \\
\text { 10. Salariés agricoles } \ldots \ldots \ldots \ldots \ldots \ldots \ldots\end{array}$ & 304 & 13 & 175 & 9 \\
\hline 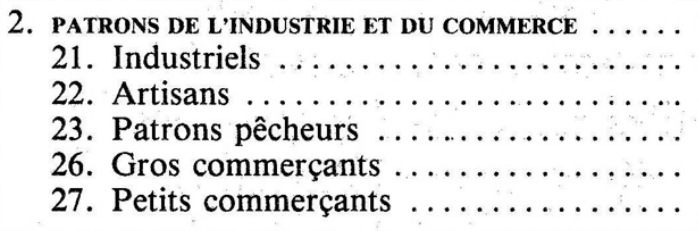 & $\begin{array}{r}1737 \\
71 \\
573 \\
13 \\
210 \\
870\end{array}$ & $\begin{array}{r}74 \\
3 \\
24 \\
1 \\
9 \\
37\end{array}$ & $\begin{array}{r}1156 \\
60 \\
464 \\
11 \\
146 \\
474\end{array}$ & $\begin{array}{r}59 \\
3 \\
24 \\
1 \\
7 \\
24\end{array}$ \\
\hline $\begin{array}{l}\text { 3. PROFESSIONS LIBÉRALES ET CADRES SUPÉRIEURS } \ldots \\
\text { 30. Professions libérales } \ldots \ldots \ldots \ldots \ldots \ldots \\
\text { 32. Professeurs, professions littéraires et } \\
\text { scientifiques } \ldots \ldots \ldots \ldots \ldots \ldots \ldots \ldots \ldots \\
\text { 33. Ingénieurs } \ldots \ldots \ldots \ldots \ldots \ldots \ldots \ldots \\
\text { 34. Cadres administratifs supérieurs } \ldots \ldots \ldots\end{array}$ & $\begin{array}{r}1810 \\
220 \\
\\
479 \\
347 \\
764\end{array}$ & $\begin{array}{r}77 \\
9 \\
20 \\
15 \\
32\end{array}$ & $\begin{array}{r}1409 \\
171 \\
292 \\
319 \\
627\end{array}$ & $\begin{array}{r}72 \\
9 \\
15 \\
16 \\
32\end{array}$ \\
\hline $\begin{array}{l}\text { 4. CADREs Movens } \ldots \ldots \ldots \ldots \ldots \ldots \ldots \\
\text { 41. Instituteurs, professions intellectuelles } \\
\text { diverses } \ldots \ldots \ldots \ldots \ldots \ldots \ldots \ldots \ldots \\
\text { 42. Services médicaux et sociaux } \ldots \ldots \ldots \ldots \\
\text { 43. Techniciens } \ldots \ldots \ldots \ldots \ldots \ldots \ldots \\
\text { 44. Cadres administratifs moyens } \ldots \ldots \ldots \ldots\end{array}$ & $\begin{array}{r}3254 \\
828 \\
432 \\
923 \\
1071\end{array}$ & $\begin{array}{l}138 \\
35 \\
18 \\
39 \\
46\end{array}$ & $\begin{array}{r}1872 \\
382 \\
170 \\
728 \\
592\end{array}$ & $\begin{array}{r}95 \\
19 \\
9 \\
37 \\
30\end{array}$ \\
\hline $\begin{array}{l}\text { 5. EMPLOÝ́s } \ldots \ldots \ldots \ldots \ldots \ldots \\
\text { 51. Employés de bureau. } \ldots \ldots \ldots \ldots \ldots \ldots \ldots \\
\text { 53. Employés de commerce } \ldots \ldots \ldots \ldots \ldots\end{array}$ & $\begin{array}{r}4677 \\
3746 \\
931\end{array}$ & $\begin{array}{r}199 \\
159 \\
40\end{array}$ & $\begin{array}{r}1882 \\
1498 \\
383\end{array}$ & $\begin{array}{l}96 \\
76 \\
20\end{array}$ \\
\hline 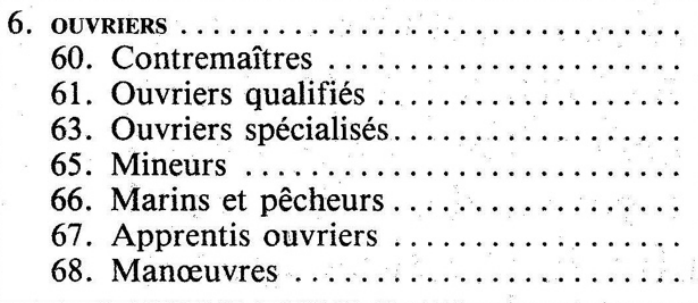 & $\begin{array}{r}8266 \\
464 \\
3292 \\
2605 \\
49 \\
33 \\
124 \\
1699\end{array}$ & $\begin{array}{r}351 \\
20 \\
140 \\
111 \\
2 \\
1 \\
5 \\
72\end{array}$ & $\begin{array}{r}5102 \\
426 \\
2336 \\
1603 \\
40 \\
25 \\
4 \\
668\end{array}$ & $\begin{array}{r}261 \\
22 \\
119 \\
82 \\
2 \\
1 \\
\mathrm{e} \\
34\end{array}$ \\
\hline $\begin{array}{l}\text { 7. PERSONNELS DE SERvice } \ldots \ldots \ldots \ldots \ldots \ldots \ldots \\
\text { 70. Gens de maison } \ldots \ldots \ldots \ldots \ldots \ldots \\
\text { 71. Femmes de ménage } \ldots \ldots \ldots \ldots \ldots \\
\text { 72. Autres personnels de service } \ldots \ldots \ldots\end{array}$ & $\begin{array}{r}1531 \\
214 \\
112 \\
1205\end{array}$ & $\begin{array}{r}65 \\
9 \\
5 \\
51\end{array}$ & $\begin{array}{r}509 \\
43 \\
22 \\
444\end{array}$ & $\begin{array}{r}26 \\
2 \\
1 \\
23\end{array}$ \\
\hline $\begin{array}{l}\text { 8. AUtres Catégories } \ldots \ldots \ldots \ldots \ldots \ldots \ldots \ldots \\
\text { 80. Artistes } \ldots \ldots \ldots \ldots \ldots \ldots \ldots \ldots \ldots \\
\text { 81. Clergé } \ldots \ldots \ldots \ldots \ldots \ldots \ldots \ldots \\
\text { 82. Armée et police } \ldots \ldots \ldots \ldots \ldots \ldots \ldots\end{array}$ & $\begin{array}{r}498 \\
74 \\
61 \\
363\end{array}$ & $\begin{array}{r}21 \\
3 \\
3 \\
15\end{array}$ & $\begin{array}{r}370 \\
49 \\
22 \\
299\end{array}$ & $\begin{array}{r}19 \\
3 \\
1 \\
15\end{array}$ \\
\hline Population active $\ldots \ldots \ldots \ldots \ldots \ldots \ldots \ldots$ & 23525 & 1000 & 13273 & 678 \\
\hline $\begin{array}{c}\text { Population inactive } \ldots \ldots \ldots \ldots \ldots \ldots \ldots \ldots \ldots \\
\text { d'âge } \quad \geqslant 15 \text { ans } \ldots \ldots \ldots \ldots \ldots \ldots \ldots \ldots \ldots \ldots\end{array}$ & $\begin{array}{ll}30 & 748 \\
19 & 515 \\
11 & 233\end{array}$ & & $\begin{array}{rr}6 & 317 \\
6 & 317 \\
& 0\end{array}$ & $\begin{array}{r}322 \\
322 \\
0\end{array}$ \\
\hline POPULATION TOTALE . & 54273 & & 19590 & 1000 \\
\hline
\end{tabular}




\section{SERMENT D'HIPPOCRATE}

Au moment d'être admis(e) à exercer la médecine, je promets et je jure d'être fidèle aux lois de l'honneur et de la probité.

Mon premier souci sera de rétablir, de préserver ou de promouvoir la santé dans tous ses éléments, physiques et mentaux, individuels et sociaux.

Je respecterai toutes les personnes, leur autonomie et leur volonté, sans aucune discrimination selon leur état ou leurs convictions. J'interviendrai pour les protéger si elles sont affaiblies, vulnérables ou menacées dans leur intégrité ou leur dignité. Même sous la contrainte, je ne ferai pas usage de mes connaissances contre les lois de l'humanité.

J'informerai les patients des décisions envisagées, de leurs raisons et de leurs conséquences.

Je ne tromperai jamais leur confiance et n'exploiterai pas le pouvoir hérité des circonstances pour forcer les consciences.

Je donnerai mes soins à l'indigent et à quiconque me les demandera. Je ne me laisserai pas influencer par la soif du gain ou la recherche de la gloire.

Admis(e) dans l'intimité des personnes, je tairai les secrets qui me seront confiés. Reçu(e) à l'intérieur des maisons, je respecterai les secrets des foyers et ma conduite ne servira pas à corrompre les moeurs.

Je ferai tout pour soulager les souffrances. Je ne prolongerai pas abusivement les agonies. Je ne provoquerai jamais la mort délibérément.

Je préserverai l'indépendance nécessaire à l'accomplissement de ma mission. Je n'entreprendrai rien qui dépasse mes compétences. Je les entretiendrai et les perfectionnerai pour assurer au mieux les services qui me seront demandés.

J'apporterai mon aide à mes confrères ainsi qu'à leurs familles dans l'adversité.

Que les hommes et mes confrères m'accordent leur estime si je suis fidèle à mes promesses ; que je sois déshonoré(e) et méprisé(e) si j'y manque.

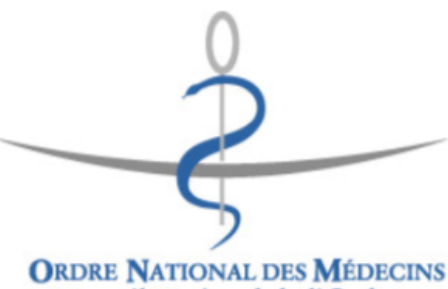


Titre : Facteurs influençant l'intérêt des patients pour l'éducation thérapeutique dans les rhumatismes inflammatoires chroniques

Auteurs : L. Reyes-Rivet (1), P. Lafforgue MD, PhD (1), T. Pham MD, PhD (1), S. Trijau MD (1)

(1) Département de Rhumatologie, Université d'Aix-Marseille, Hôpital de Sainte-Marguerite, Marseille, France.

Cher éditeur,

L'éducation thérapeutique (ETP) est une pratique recommandée par les sociétés savantes [17,35] dans l'accompagnement des soins des patients atteints de rhumatisme inflammatoire chronique (RIC).

Dans les RIC, l'ETP a montré des bénéfices sur la douleur, les connaissances sur la maladie, les comportements d'adaptation à court terme, le soutien social $[20,23]$ ainsi que des effets à long terme sur l'activité de la maladie [24]. Cependant, les mises en route de programme d'ETP nécessitent un investissement conséquent pour les soignants qui les organisent et les patients qui y participent. Il est donc important, pour mieux optimiser l'adhésion à l'ETP de savoir à qui le proposer. Or si les besoins éducationnels des patients ont été bien évalués [27], très peu de données nous renseignent sur les profils de patients intéressés par l'ETP.

Nous avons cherché à identifier les patients intéressés par l'ETP.

Les patients atteints de RIC (polyarthrite rhumatoïde, rhumatisme psoriasique ou spondyloarthrite) vus en hôpital de jour (HDJ) ou en consultation au CHU de 
Marseille ont été inclus sur une durée de quatre semaines consécutives. Il leur était proposé de visionner une vidéo d'explication sur l'ETP (annexe 1) puis de répondre à un questionnaire (annexe 2). La question était « seriez-vous intéressé par le programme d'ETP ?», et nous avons recherché quels paramètres étaient associés à cette réponse. Les données démographiques, concernant la maladie, socioprofessionnelles étaient collectées sur le dossier médical informatisé.

Parmi les 183 patients inclus entre septembre et octobre 2019, 143 ont répondu à la question sur l'intérêt pour l'ETP.

En analyses univariées (tableau 1), un diagnostic inférieur à 5 ans et le recrutement en consultation était significativement associé à l'intérêt pour l'ETP (respectivement $O R=5,456 ; p=0,024$ ) et $O R=7,562 ; p=0,007)$. La catégorie professionnelle influence l'intérêt pour l'ETP.

Sur les analyses multivariées, seule la modalité de proposition est statistiquement significatif, indépendamment associé à l'intérêt pour l'ETP (OR $=2,275(1,077 ; 4,810) ; p=0,031)$ (Table 2).

Nous avons trouvé qu'un âge plus jeune, un diagnostic récent et une proposition au cours d'une consultation sont des critères d'intérêt à l'éducation thérapeutique. Le fait que l'intérêt pour la démarche d'ETP soit moindre dans la population suivie en HDJ peut refléter le fait qu'elle se sent suffisamment encadrée par l'équipe médicale, par rapport à une population purement ambulatoire se sentant livrée à elle-même. Inversement, certains facteurs ne sont pas associés à cet intérêt, notamment le type de rhumatisme inflammatoire, l'activité de la maladie ou l'incidence d'effet indésirable lié au traitement dans l'année écoulée. Ces deux derniers sont probablement liés à notre population : nous avions en effet peu de patients avec une maladie très active ou ayant eu des événements indésirables. 
L'éducation thérapeutique, pour être optimale, doit être proposée au bon moment et au bon patient. D'autres études sont nécessaires pour confirmer ces données mais il semblerait que les patients jeunes au début de leur prise en charge soient des patients cibles pour l'ETP. Une consultation dédiée à l'annonce de l'ETP semblerait pertinente. 
Tableau 1 : Caractéristiques des patients en fonction de leur intérêt pour l'ETP

\begin{tabular}{|c|c|c|c|c|c|}
\hline & & & & & \\
\hline & & \multicolumn{2}{|l|}{ Intérêt ETP } & \multirow[t]{2}{*}{$\mathrm{OR}^{*}$} & \multirow[t]{2}{*}{$p$} \\
\hline & & Non $(n=70)$ & $\begin{array}{l}\text { Oui } \\
(n=73)\end{array}$ & & \\
\hline \multirow[t]{3}{*}{ Pathologie } & SPA, n (\%) & $31(47,7)$ & $34(52,3)$ & 2.616 & 0.274 \\
\hline & $\mathrm{PR}, \mathrm{n}(\%)$ & $25(58,1)$ & $18(41,9)$ & & - \\
\hline & Rh Pso, n (\%) & $14(40,0)$ & $21(60,0)$ & & - \\
\hline \multirow{2}{*}{$\begin{array}{l}\text { Date du diagnostic } \\
\text { (an) }\end{array}$} & $<5, \mathrm{n}(\%)$ & $9(30,0)$ & $21(70,0)$ & 5.456 & 0.024 \\
\hline & $>5 n(\%)$ & $61(54,0)$ & $52(46,0)$ & & - \\
\hline \multirow[t]{2}{*}{ Activité de la maladie } & $\begin{array}{l}\text { Rémission/faible, } \\
\mathrm{n}(\%)\end{array}$ & $47(52.8)$ & $42(47.2)$ & 2.469 & 0.162 \\
\hline & Forte, n (\%) & $13(37.1)$ & $22(62.9)$ & & \\
\hline \multirow[t]{2}{*}{ Biothérapie } & Oui, n (\%) & $\begin{array}{l}59 \\
(51.3)\end{array}$ & $56(48.7)$ & 0.438 & 0.660 \\
\hline & Non, n (\%) & $\begin{array}{l}11 \\
(44.0)\end{array}$ & $14(56.0)$ & & \\
\hline \multirow[t]{2}{*}{$\begin{array}{l}\text { Modalités de } \\
\text { proposition }\end{array}$} & HDJ, n (\%) & $\begin{array}{l}39 \\
(61.9)\end{array}$ & $24(38.1)$ & 7.562 & 0.007 \\
\hline & $\begin{array}{l}\text { Consultation, } n \\
(\%)\end{array}$ & $\begin{array}{l}31 \\
(38.8)\end{array}$ & $49(61.3)$ & & \\
\hline \multirow[t]{2}{*}{ Sexe } & Homme, n (\%) & $\begin{array}{l}24 \\
(53.3)\end{array}$ & $21(46.7)$ & 0.505 & 0.589 \\
\hline & Femme, n (\%) & $\begin{array}{l}46 \\
(49.9)\end{array}$ & $52(53.1)$ & & \\
\hline \multirow[t]{3}{*}{ Niveau d'éducation } & Brevet & $18(45.0)$ & $22(55.0)$ & 1.434 & 0.489 \\
\hline & $\mathrm{Bac}$ & $24(53.3)$ & $21(46.7)$ & & \\
\hline & Supérieur & $18(40.9)$ & $26(59.1)$ & & \\
\hline \multirow[t]{2}{*}{ Langue maternelle } & Française & $61(49.2)$ & $63(50.8)$ & 0.034 & 1 \\
\hline & Autres & $7(46.7)$ & $8(53.3)$ & & \\
\hline En activité & Oui, n (\%) & $29(42.6)$ & $39(57.4)$ & 1.615 & 0.135 \\
\hline Catégories & Sans & $8(72.7)$ & $3(27.3)$ & 15.275 & 0.015 \\
\hline \multirow[t]{6}{*}{ socioprofessionnelles } & 1 & $19(59.4)$ & $13(40.6)$ & - & - \\
\hline & 2 & $3(50.0)$ & $3(50.0)$ & - & - \\
\hline & 3 & $15(45.5)$ & $18(54.5)$ & - & - \\
\hline & 4 & $7(35.0)$ & $13(65.0)$ & - & - \\
\hline & 5 & $10(31.3)$ & $22(68.8)$ & - & - \\
\hline & 6 & $8(88.9)$ & $1(11.1)$ & - & - \\
\hline $\begin{array}{l}\text { Effets indésirables < } \\
12 \text { mois }\end{array}$ & Oui, n (\%) & $25(53.2)$ & $22(46.8)$ & 0.358 & 0.593 \\
\hline
\end{tabular}

HDJ = Hôpital de jour 
Tableau 2 : Analyse de régression logistique multivariée des facteurs associés à l'intérêt à l'éducation thérapeutique.

OR univarié $\quad \mathrm{p} \quad$ OR multivarié (IC 95\%) $\mathrm{p}$

\begin{tabular}{lcccc}
\hline Ancienneté du diagnostic & 5,545 & 0,024 & $0,03(0,150 ; 1,081)$ & 0,071 \\
\hline $\begin{array}{l}\text { Lieu de proposition } \\
\text { Consultation vs HDJ }\end{array}$ & 7,562 & 0.007 & $2,275(1,077 ; 4,810)$ & 0,031 \\
\hline En activité (professionnelle) & 1,615 & 0,135 & $1,482(0,692 ; 3,171)$ & 0,311 \\
& & & & \\
\hline Activité de la maladie & 2.469 & 0.162 & $1,550(0,664 ; 3,617)$ & 0,311
\end{tabular}

\section{REFERENCES}

[1] Daien C, Hua C, Gaujoux-Viala C, Cantagrel A, Dubremetz M, Dougados M, et al. Actualisation des Recommandations de la Société française de rhumatologie pour la prise en charge de la polyarthrite rhumatoïde. Rev Rhum 2019;86:8-24. https://doi.org/10.1016/j.rhum.2018.09.008.

[2] Combe B, Landewe R, Daien Cl, Hua C, Aletaha D, Álvaro-Gracia JM, et al. 2016 update of the EULAR recommendations for the management of early arthritis. Ann Rheum Dis 2017;76:948-59. https://doi.org/10.1136/annrheumdis-2016-210602.

[3] Stenberg U, Haaland- $\varnothing$ verby M, Fredriksen K, Westermann KF, Kvisvik T. A scoping review of the literature on benefits and challenges of participating in patient education programs aimed at promoting self-management for people living with chronic illness. Patient Educ Couns 2016;99:1759-71. https://doi.org/10.1016/j.pec.2016.07.027.

[4] Niedermann K, Fransen J, Knols R, Uebelhart D. Gap between short- and long-term effects of patient education in rheumatoid arthritis patients: A systematic review: Patient Education in RA Patients. Arthritis Care Res 2004;51:388-98. https://doi.org/10.1002/art.20399.

[5] Abourazzak F, El Mansouri L, Huchet D, Lozac'hmeur R, Hajjaj-Hassouni N, Ingels A, et al. Long-term effects of therapeutic education for patients with rheumatoid arthritis. Joint Bone Spine 2009;76:648-53. https://doi.org/10.1016/j.jbspin.2009.01.010.

[6] Perdriger A, Pouplin S, Dartois A, Beauvais C. Objectifs et besoins éducatifs des patients atteints de rhumatismes inflammatoires chroniques. Rev Rhum Monogr 2013;80:152-6. https://doi.org/10.1016/j.monrhu.2013.04.007. 